\title{
Gradient-based design of actively-cooled microvascular composite panels
}

\author{
Marcus Hwai Yik Tan ${ }^{\mathrm{a}, \mathrm{d}}$, Ahmad R. Najafi,d, Stephen J. Pety ${ }^{\mathrm{b}, \mathrm{d}}$, Scott R. \\ White $^{\mathrm{c}, \mathrm{d}}$, Philippe H. Geubelle $\mathrm{e}^{\mathrm{c}, \mathrm{d}, 1}$ \\ ${ }^{a}$ Department of Mechanical Science and Engineering, University of Illinois, Urbana, IL 61801, \\ USA \\ ${ }^{b}$ Department of Material Science and Engineering, University of Illinois, Urbana, IL 61801, USA \\ ${ }^{c}$ Department of Aerospace Engineering, University of Illinois, Urbana, IL 61801, USA \\ ${ }^{d}$ Beckman Institute for Advanced Science and Technology, University of Illinois, Urbana, IL \\ 61801, USA
}

\begin{abstract}
Recent advances in manufacturing based on sacrificial fiber or template techniques have allowed complex networks of microchannels to be embedded in microvascular composites. In the thermal application of interest, a novel battery packaging scheme for electric vehicles is considered where each battery is surrounded by microvascular composite panels for temperature regulation and structural protection. We use simplified thermal and hydraulics models validated against more complex 3D FLUENT simulations and experiments to obtain the surface temperature distribution of the panel and the pressure drops across the microchannels. We further eliminate the cost and complexity associated with mesh generation by applying the interface-enriched generalized finite element method (IGFEM), which allows a non-conforming mesh to capture the discontinuous temperature gradient across the microchannels. The IGFEM thermal solver is then combined with a gradient-based shape optimization scheme to obtain optimal designs of a set of branched microchannel networks. The design parameters are the channel control points, which define the shape of the network. We use the $p$-mean as a differentiable objective function in place of the maximum temperature. To obtain accurate gradients with respect to the design parameters efficiently, we perform a sensitivity analysis based on a recently developed adjoint method for IGFEM. Starting from many distinct configurations, we obtain the optimal designs for a wide range of network topologies. We also investigate the
\end{abstract}

Email address: geubelle@illinois.edu (Philippe H. Geubelle)

${ }^{1}$ Corresponding author. geubelle@illinois.edu. Tel: +1-217-244-7648. Fax: +1-217-244 0720

Preprint submitted to Int. J. Heat Mass Transfer

July 20, 2016

(C) 2016. This manuscript version is made available under the Elsevier user license

http://www.elsevier.com/open-access/userlicense/1.0/ 
effect of the coolant flow rate on the optimal design.

Keywords:

battery cooling, optimization, interface-enriched generalized finite element method, microvascular composite, simplified thermal model, microchannel

\section{Introduction}

Microvascular composites are a novel class of biomimetic materials that contain embedded microchannels resembling vascular systems found in nature. Recently introduced sacrificial fiber and template manufacturing techniques have allowed for the embedding of complex networks of microchannels in composite materials $[1,2]$. The resulting material can be used for multifunctional applications based on the choice of fluid present in the microchannels. For example, one can change the electromagnetic signature by using a ferrofluid, tune the electrical properties by using an electrically conductive fluid, enable self-healing of the material by allowing appropriate chemicals to flow to damage sites, and regulate the temperature of the material by circulating coolants $[1,3,4,5]$. The thermal applications include space reentry or hypersonic vehicles, high-power electronics cooling and car battery cooling $[6,7,8,9,10,11]$. While this work focuses on battery cooling applications, the method described here can be applied to other materials with embedded microchannels used for thermal management.

Battery cooling is essential to extend the range of electric vehicles and prolong battery life [12]. For batteries with high energy density such as those in electric vehicles, liquid cooling is the most effective cooling method [13]. A typical battery packaging for electric vehicles consists of stacks of battery cells separated by fiberglass or steel panels to provide structural protection and cooling plates to regulate battery temperature. Recently, a novel battery packaging scheme in which a single microvascular composite provides both cooling and crash protection has been proposed [11]. The crash protection is superior to conventional battery packaging because the carbon fiber reinforced composite possesses high specific strength, stiffness as well as energy absorbing ability [14, 15].

The performance of battery cooling panels is usually analyzed using a fluidthermal solver that combines the Navier-Stokes and energy equations $[16,17]$. Because the Navier-Stokes equations are nonlinear, the coupled fluid-thermal problem has to be solved iteratively until the residuals of the equations fall below a userdefined tolerance. To solve the equations, a sufficiently refined mesh aligned with the walls of the embedded channels has to be generated. Due to the large disparity in length scales between a microchannel diameter and the panel dimensions, many 
elements have to be created to ensure that the growth in element size from the interior of a microchannel to the domain boundary does not result in elements with high aspect ratios. As a result, a full-blown coupled fluid/thermal analysis of the microvascular panels is computationally expensive. This is especially the case when numerous designs have to be evaluated in an optimization or parametric study.

Therefore, in this work, we use a simplified thermal model based on a simple energy balance of an infinitesimal segment of a channel. The simple model only requires the solution of a linear heat equation with an extra source/sink term due to the microchannels. Moreover, because the microchannels are collapsed into lines or curves, the mesh generation burden is greatly reduced. By verifying the model with the commercial software ANSYS FLUENT v15.0 and validating the model with experiments, we show that this simple model is sufficiently accurate for the battery cooling application.

We further improve efficiency by using the interface-enriched generalized finite element method (IGFEM) [18, 19], which allows capturing the discontinuous temperature gradient across the microchannels with a nonconforming mesh, thus eliminating the mesh generation complexity and cost. Due to the use of stationary and non-conforming mesh, a key advantage of the IGFEM, generalized/extended finite element method (GFEM/XFEM) and other Eulerian approaches over the standard FEM (SFEM) in shape optimization analysis is that they do not suffer from the severe mesh distortion that the SFEM often faces when the shape evolves [20, 21]. Another key advantage is that only the nodal velocities of the enrichment nodes along the microchannels need to be evaluated, thus limiting the computational work for the sensitivity analysis to the enriched elements intersecting the microchannels.

Because the design of the embedded channels is a crucial factor in the performance of cooling panels, it has been the subject of multiple studies. A performance evaluation of different base channel designs such as parallel, bifurcating/tree-like, serpentine, spiral, coiled, bifurcating-parallel hybrid and other designs was presented in $[17,22,23,24]$. Within a base design, we can further optimize the performance by changing the shape of the channels. Due to the large number of associated design parameters, gradient-based shape optimization [16] is more tractable than parametric studies [25]. Other optimization methods that do not require a starting base design are topology optimization [26], discrete topology optimization connecting lattice points in space $[27,28,29]$ or constructal theory [30]. It is also worth mentioning that optimization methods have also been applied to microchannel heat sink for microelectronics cooling, but with a typical topology of unconnected channels running parallel to each other $[31,32]$. In these studies, the design parameters are usually channel dimensions and spacing between channels. 
In optimization studies of cooling panels, objective functions commonly considered are average temperature and standard deviation, a measure of temperature uniformity [16, 26]. Temperature uniformity is important because non-uniformity causes variations in reaction rates that lead to incomplete energy utilization and shorter battery life [12]. However, average temperature alone as an objective function is not sufficient to keep the maximum temperature in the panel low. Furthermore, as seen later, the average temperature is relatively insensitive to the channel design. Besides, small regions with high temperature akin to the appearance of areas with stress concentration in a compliance-based optimization [33] may also appear in the optimal design. This suggests that the maximum temperature be minimized rather than the average temperature. Unfortunately, the maximum temperature is not differentiable in a classical sense [34] and therefore conventional gradient-based optimizer cannot be used when the maximum temperature is chosen as the objective function. Instead, following a standard practice in stress-based optimization [35, 36, 33], the $p$-mean of the temperature with a sufficiently large $p$ is used in this work.

The paper is organized as follows: In Section 2, we introduce the simplified thermal model, the IGFEM solver and a Streamline Upwind/Petrov-Galerkin (SUPG) scheme employed to stabilize the IGFEM solution. We then explain in Section 3 the hydraulics equations used to model the pressure drops and flow rates in the microchannel network. The numerical model is verified against more complex 3D, fully coupled FLUENT simulations and validated against experiments in Section 4. We then describe the general optimization problem and the sensitivity analysis relevant to this work in Section 5. Lastly, we apply the IGFEM-based shape optimization scheme to optimize different parallel network designs defined by the number of branches and validate one of the optimized designs in Section 6.

\section{Simplified Model and Interface-Enriched Generalized Finite Element Method}

While prior works $[25,37,8]$ model the microchannels with finite diameters, we take advantage in the present study of the low diameter-to-length ratio of a microchannel to collapse the microchannels into line sources/sinks [38, 28, 29, 39]. In the case of non-circular cross section, "diameter" refers to the largest dimension of the cross section.

For brevity, we use hereafter the word "channel" to refer to a microchannel. Consider a channel with cross-sectional area $A$, axial velocity $u$ and average velocity $u_{\text {ave }}$. Let $T_{m}$ be the mixed-mean fluid temperature, defined as $T_{m}=\int u T \mathrm{~d} A /\left(A u_{\text {ave }}\right)$ [40]. Further, let $s, \dot{m}$ and $c_{f}$ respectively denote the parametric coordinate along 
the channel in the flow direction, the mass flow rate and the specific heat capacity of the fluid. A simple energy balance over an infinitesimal portion of a channel yields the following expression for the heat flow rate per unit length of the channel [40]:

$$
q^{\prime}=\dot{m} c_{f} \frac{\mathrm{d} T_{m}}{\mathrm{~d} s} .
$$

Let us further assume that $T_{m}$ is approximately equal to its wall temperature, $T_{w}$. Given the thermal conductivity tensor $\boldsymbol{\kappa}$ of the solid, a distributed heat source $f(\mathbf{x})$, the convection coefficient $h$ and an ambient temperature $T_{\mathrm{amb}}$, the heat equation with contribution from $n_{c h}$ channels is given by

$$
\nabla \cdot(\boldsymbol{\kappa} \nabla T)+f(\mathbf{x})=\sum_{i=1}^{n_{c h}} \delta_{l}^{(i)}(\mathbf{x}) \gamma^{(i)} \boldsymbol{t}^{(i)} \cdot \nabla T+h\left(T-T_{\mathrm{amb}}\right),
$$

where $\gamma^{(i)}=\dot{m}^{(i)} c_{f}, \boldsymbol{t}^{(i)}$ is the unit tangent vector of channel $i$ in the flow direction and $\delta_{l}^{(i)}(\mathbf{x})=\int_{l} \delta\left(\mathbf{x}-\mathbf{x}^{(i)}(s)\right) \mathrm{d} s$ is the line Dirac delta function associated with channel $i$. The assumptions made to derive this equation are discussed in Appendix I.

Let the boundary of domain $\Omega$ be divided into two parts, $\Gamma_{T}$ and $\Gamma_{q}$, where Dirichlet and Neumann boundary conditions are specified, respectively. Denoting channel $i$ by $\Gamma_{f}^{(i)}$ and the prescribed heat flux as $q_{p s}^{\prime \prime}$, the weak form of $(2)$ is: Find the temperature field $T$ satisfying the Dirichlet boundary condition $T_{\mid \Gamma_{T}}=T_{p s}$ such that $\forall v \in \mathbb{V}$,

$$
\begin{aligned}
0= & -\int_{\Omega_{s}}(\nabla v \cdot \boldsymbol{\kappa} \nabla T+h v T) \mathrm{d} \Omega-\sum_{i=1}^{n_{c h}} \int_{\Gamma_{f}^{(i)}} v \gamma^{(i)} \boldsymbol{t}^{(i)} \cdot \nabla T \mathrm{~d} \Gamma \\
& +\int_{\Omega_{s}} v\left(f+h T_{\mathrm{amb}}\right) \mathrm{d} \Omega+\int_{\Gamma_{q}} v q_{p s}^{\prime \prime} \mathrm{d} \Gamma,
\end{aligned}
$$

where $\mathbb{V}$ is the space of weighing functions. The presence of the convective term in (3) implies that the associated stiffness matrix is not symmetric. For a single channel, this term only causes instability at very high flow rates, well beyond the regime of interest (below $60.0 \mathrm{ml} / \mathrm{min}$ ). However, when multiple channels with branching in the domain are present, we observe numerical oscillations in the solution similar to those that appear when modeling the channels with non-zero diameters [37] at the flow rates of interest in this study.

To remove the oscillations, we employ the streamline upwind/Petrov-Galerkin (SUPG) method [41], which modifies the weighing function while maintaining equivalence with the original weak form. As only balanced plain-weave composites are 
considered in this work, the in-plane conductivity is assumed to be isotropic [42] and we write the $2 \mathrm{D}$ conductivity tensor as $\kappa=\kappa \mathbf{I}$. Denoting the density of the fluid by $\rho_{f}$, the total number of the nodes in the mesh by $n_{n}$ and the shape function associated with node $j$ by $N_{j}$, we replace $v$ in (3) by

$$
w=v+\sum_{i=1}^{n_{c h}} \tau_{e}^{(i)} u_{\mathrm{ave}}^{(i)} \boldsymbol{t}^{(i)} \cdot \nabla v
$$

where

$$
\tau_{e}^{(i)}=\frac{h_{e}^{(i)}}{2 u_{\mathrm{ave}}^{(i)}}\left[\operatorname{coth}\left(\frac{u_{\mathrm{ave}}^{(i)} h_{e}^{(i)} \rho_{f} c_{f}}{2 \kappa}\right)-\frac{2 \kappa}{u_{\mathrm{ave}}^{(i)} h_{e}^{(i)} \rho_{f} c_{f}}\right],
$$

and

$$
\frac{2}{h_{e}^{(i)}}=\sum_{j=1}^{n_{n}}\left|\boldsymbol{t}^{(i)} \cdot \nabla_{x} N_{j}\right|
$$

Note that the streamline upwind contribution (second term of (4)) is understood to vanish at the boundaries [41]. Because linear triangular elements are used in this work, $\nabla_{x x} v=0$, which implies that $\nabla_{x} w=\nabla_{x} v$. Since our simplified model depends explicitly on $\dot{m}^{(i)}$ rather than $u_{\text {ave }}^{(i)}$, and $u_{\text {ave }}^{(i)}$ arbitrarily depends on the assumed cross-sectional area, we eliminate $u_{\text {ave }}^{(i)}$ from (5) by assuming $u_{\text {ave }}^{(i)} h_{e}^{(i)} \rho c_{f} / \kappa \gg 1$. This assumption is valid because $u_{\text {ave }}^{(i)} h_{e}^{(i)} \rho c_{f} / \kappa \approx 7000$, based on the parameters used in the simulations described in Section 4. Therefore, (5) simplifies to $\tau_{e}^{(i)}=h_{e}^{(i)} /\left(2 u_{\mathrm{ave}}^{(i)}\right)$ and (4) becomes

$$
w=v+\sum_{i=1}^{n_{c h}} \frac{h_{e}^{(i)}}{2} \boldsymbol{t}^{(i)} \cdot \nabla_{x} v .
$$

In the finite element method, the weak form in (3) is solved by approximating $\mathbb{V}$ by a finite dimensional space. Let there be $n_{\text {on }}$ original nodes in the non-conforming mesh with $u_{i}$ and $N_{i}(\mathbf{x})$ respectively denoting the nodal value and the Lagragian shape function associated with node $i$. In the IGFEM formulation $[18,19], n_{e n}$ enrichment nodes are added along the channels to yield the following appproximation to the temperature field:

$$
T^{h}(\mathbf{x})=\sum_{i=1}^{n_{\text {on }}} u_{i} N_{i}(\mathbf{x})+\sum_{j=1}^{n_{\text {en }}} \beta_{j} \psi_{j}(\mathbf{x})=\left\{\begin{array}{l}
\{N(\mathbf{x})\} \\
\{\psi(\mathbf{x})\}
\end{array}\right\}^{\prime}\left\{\begin{array}{l}
\{T\} \\
\{\beta\}
\end{array}\right\},
$$

where $\psi_{j}$ is the enrichment function associated with enrichment node $j, \beta_{j}$ is the generalized degree of freedom and $\{\cdot\}^{\prime}$ denotes the transpose of the vector $\{\cdot\}$. For 
simplicity in presenting the adjoint method in Section 5, we hereafter omit the distinction between original and enrichment degrees of freedom as well as between original shape functions and enrichment functions, and write (8) as:

$$
T^{h}(\mathbf{x})=\{N(\mathbf{x})\}^{\prime}\{T\} .
$$

From (7), the SUPG weighing function has the form

$$
w^{h}(\mathbf{x})=\{W(\mathbf{x})\}^{\prime}\{V\}
$$

where

$$
\{W\}=\{N\}+\sum_{i=1}^{n_{c h}} \frac{h_{e}^{(i)}}{2}[B]\left\{t^{(i)}\right\}
$$

and $[B]$ is the matrix of the spatial derivative of $\{N\}$ with each column corresponding to the derivative with respect to a coordinate. The second term of (11) is understood to vanish at element boundaries [41].

Due to the arbitrariness of the weighing function, the discretized weak form reduces to

$$
[K]\{T\}=\{F\}
$$

where

$$
[K]=\int_{\Omega_{s}}\left([B][\kappa][B]^{\prime}+h\{W\}\{N\}^{\prime}\right) \mathrm{d} \Omega+\sum_{i=1}^{n_{c h}} \int_{\Gamma_{f}^{(i)}}\{W\}\left([B] \gamma^{(i)}\left\{t^{(i)}\right\}\right)^{\prime} \mathrm{d} \Gamma
$$

and

$$
\{F\}=\int_{\Omega_{s}}\{W\}\left(f+h T_{\mathrm{amb}}\right) \mathrm{d} \Omega+\int_{\Gamma_{q}}\{V\} q_{\mathrm{ps}}^{\prime \prime} \mathrm{d} \Gamma .
$$

Details on the assembling of the stiffness matrix $[K]$ and the load vector $\{F\}$ can be found in [21].

\section{Hydraulics Equations}

To obtain the mass flow rate in channel $i, \dot{m}^{(i)}$, we first solve for the pressure $P_{j}$ at each end point or node of the channel network using a system of hydraulics equations. The equations are assembled from the following relation between the

nodal pressures, $P_{j}, P_{k}$ of channel $i$ and the contribution of its flow rate $S_{j}^{(i)}, S_{k}^{(i)}$ to the nodes $j, k[43]$ :

$$
g^{(i)}\left[\begin{array}{cc}
1 & -1 \\
-1 & 1
\end{array}\right]\left\{\begin{array}{l}
P_{j} \\
P_{k}
\end{array}\right\}=\left\{\begin{array}{l}
S_{j}^{(i)} \\
S_{k}^{(i)}
\end{array}\right\}
$$


where $g^{(i)}$ is the conductance of channel $i$. For a square cross section of width $D$ or a circular cross section of diameter $D$, a channel length $L$ and a kinematic viscosity of $\nu$, the conductance is given by [44]

$$
g=\frac{k D^{4}}{\nu L}
$$

For square cross sections, $k=1 / 28.46$ while for circular cross sections, $k=\pi / 128 \approx$ $1 / 40.74[44,45]$. For rectangular cross sections, $k D^{4}$ is replaced by $a^{3} b\{1 / 3-$ $\left.64 a \tanh [\pi b /(2 a)] /\left(4 \pi^{5} b\right)\right\}$, where $a, b$ respectively denote the height and width of a cross section [44]. If $P_{j}$ and $P_{k}$ are the pressures at the two nodes of channel $i$, then $\dot{m}^{(i)}=g^{(i)}\left|P_{j}-P_{k}\right|$.

All properties are assumed to be independent of temperature, except for the dynamic viscosity of the fluid (in Pa.s), given by [46]

$$
\mu(T)=0.0069\left(\frac{T}{273.15}\right)^{-8.3},
$$

where the temperature $T$ is expressed in $\mathrm{K}$. The dynamic viscosity used for the hydraulics equations is evaluated at the average temperature of the domain, $\langle T\rangle=$ $\int_{\Omega} T \mathrm{~d} \Omega /|\Omega|$.

\section{Validation Study}

Experiments were performed on microvascular composite panels with the setup shown in Fig. 1a and parameters presented in Table 1. A composite panel, shown schematically in Fig. 2a, was heated from below by an electric heater with a heat distribution profile $f(\mathbf{x})=f(x, y)$ shown in Fig. 1b and with average heat flux characteristic of that generated by adjacent battery pouches, i.e., $\int_{\Omega} f(x, y) \mathrm{d} \Omega /|\Omega|=$ $500 \mathrm{~W} / \mathrm{m}^{2}$. A water ethylene/glycol coolant was pumped through the vascular network with a peristaltic pump and the resulting pressure drop across the channel network was measured with pressure transducers. While the inlet temperature was determined with a thermocouple, the temperature distribution of the top surface of the panel was recorded with an infrared (IR) camera.

We apply $f(x, y)$ on the bottom face, allow convective and radiative heat loss from the top face and insulate the thin sides of the panel as shown in Fig. 2a and 2b to simulate the conditions of the experiments in FLUENT. The convective heat loss is modeled as $q_{\text {conv }}^{\prime \prime}=-h_{\text {conv }}\left(T-T_{\mathrm{amb}}\right)$, where $h_{\text {conv }}$ is found by fitting simulation results to results from experiments $[10,11]$. Using the Stefan-Boltzmann law for gray surfaces, the radiative heat loss is modeled as $q_{\mathrm{rad}}^{\prime \prime}=-\epsilon \sigma_{B}\left(T^{4}-T_{\mathrm{amb}}^{4}\right)$, where $\sigma_{B}$ is 


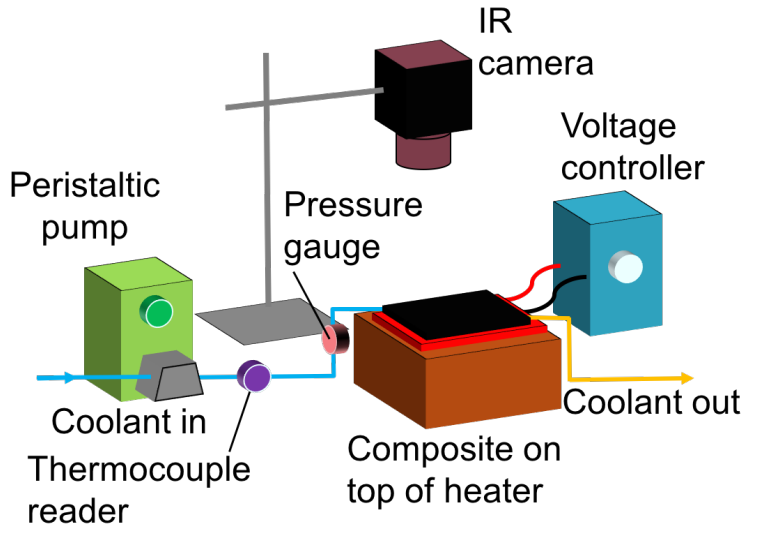

(a) Experimental setup.

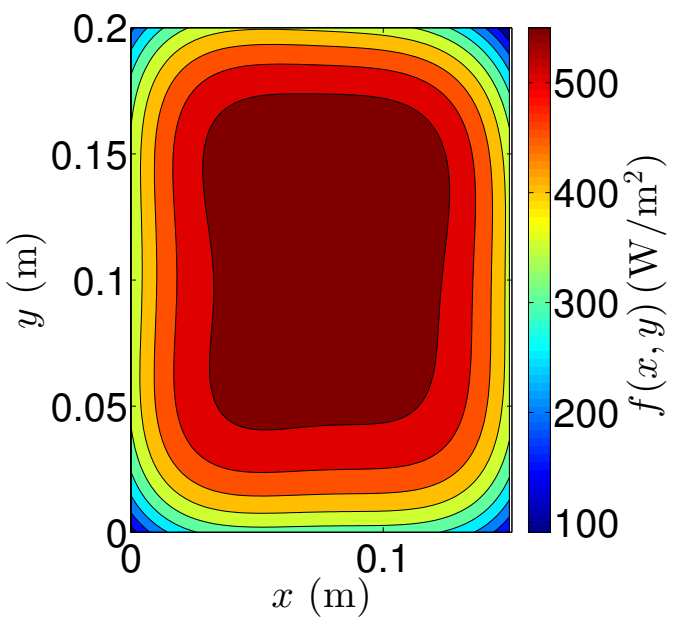

(b) Experimental heat source.

Figure 1: (a) Experimental setup for the heating of a microvascular composite panel with activecooling .(b) The fourth-order polynomial fit, $f(x, y)=500\left(0.2489+23.42 x+21.87 y-485.7 x^{2}-\right.$ $16.96 x y-356.8 y^{2}+4317 x^{3}+170.4 y x^{2}+276.7 y^{2} x+2422 y^{3}-14060 x^{4}-791 y x^{3}-490 x^{2} y^{2}-972.2 y^{3} x-$ $\left.5849 y^{4}\right) \mathrm{Wm}^{-2}$, to the heat flux distribution of the heater.

Table 1: Experimental conditions, coolant and composite thermal properties.

\begin{tabular}{|c|c|}
\hline In-plane dimension, $L_{x}(\mathrm{~mm})$ & 150 \\
\hline In-plane dimension, $L_{y}(\mathrm{~mm})$ & 200 \\
\hline Panel thickness, $t(\mathrm{~mm})$ & 3 \\
\hline Fin coefficient, $h_{\text {conv }}\left(\mathrm{Wm}^{-2} \mathrm{~K}^{-1}\right)$ & 8 \\
\hline Ambient temperature, $T_{\mathrm{amb}}\left({ }^{\circ} \mathrm{C}\right)$ & 21 \\
\hline Emissivity, $\epsilon$ & 0.97 \\
\hline Coolant density $\left(\mathrm{kgm}^{-3}\right)$ & 1065 \\
\hline Coolant conductivity $\left(\mathrm{Wm}^{-1} \mathrm{~K}^{-1}\right)$ & 0.419 \\
\hline Coolant heat capacity $\left(\mathrm{Jkg}^{-1} \mathrm{~K}^{-1}\right)$ & 3494 \\
\hline Coolant flow rate $\left(\mathrm{ml} \mathrm{min}^{-1}\right)$ & 28.2 \\
\hline Coolant inlet temperature $\left({ }^{\circ} \mathrm{C}\right)$ & 22 \\
\hline In-plane conductivity $\left(\mathrm{Wm}^{-1} \mathrm{~K}^{-1}\right)$ & 2.04 \\
\hline Out-of-plane conductivity $\left(\mathrm{Wm}^{-1} \mathrm{~K}^{-1}\right)$ & 0.59 \\
\hline
\end{tabular}

the Stefan-Boltzmann constant. The emissivity of the matte black paint used on the panels, $\epsilon$ is provided by the vendor.

The thermal conductivities of the composite were calculated basd on the measured 


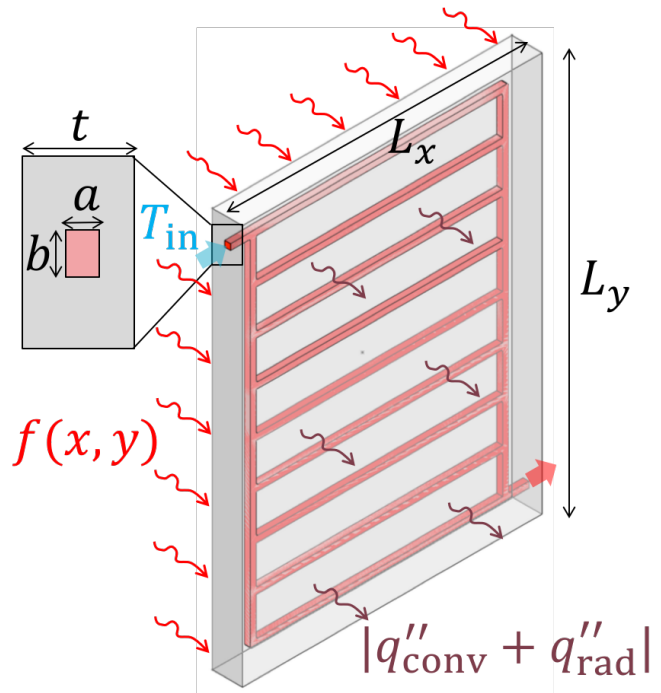

(a)

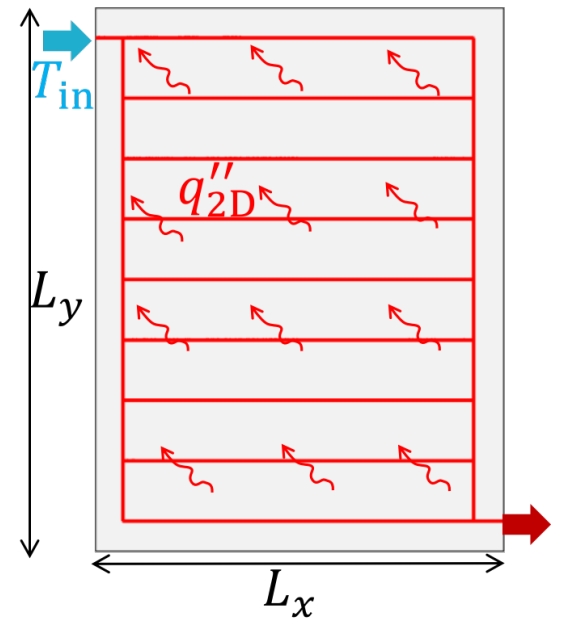

(b)

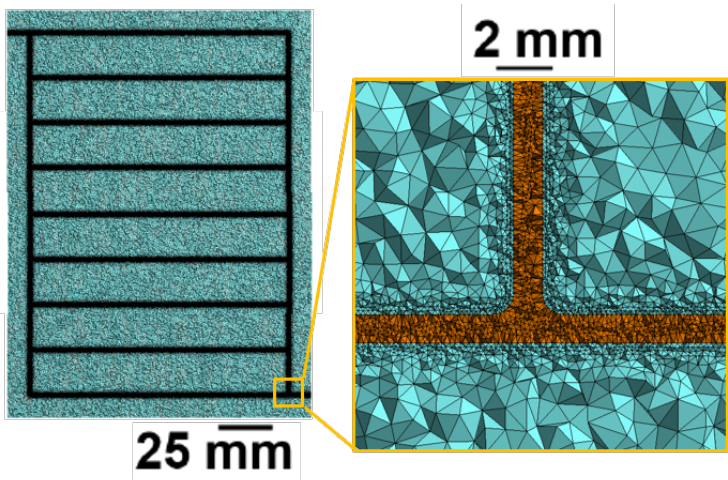

(c)
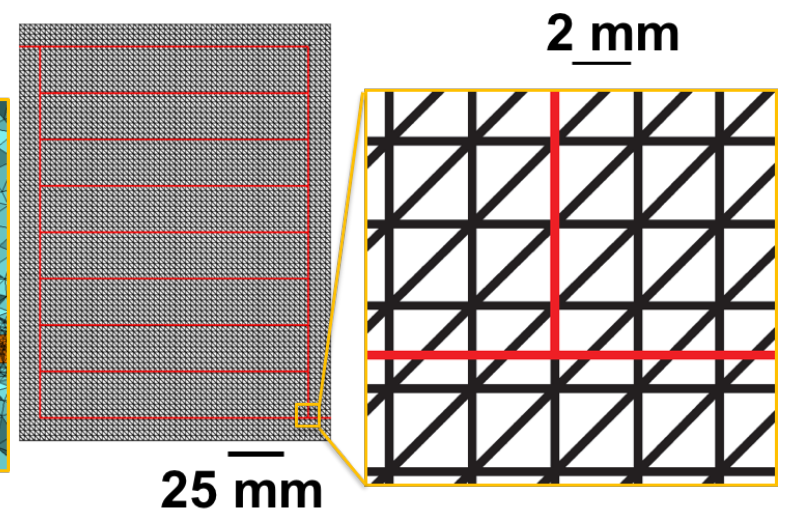

(d)

Figure 2: (a) FLUENT setup and (b) IGFEM setup with $q_{2 \mathrm{D}}^{\prime \prime}=f(x, y)-h\left(T-T_{\mathrm{amb}}\right)$ for validation. Meshes used for the (c) FLUENT and (d) IGFEM simulations.

volume fraction of fiber (45\%) and knowledge of thermal conductivities of the carbon fiber and epoxy as provided by the manufacturer. The in-plane conductivity was then calculated using a closed-form solution developed in [42] and the out-of-plane conductivity was calculated using a self-consistent model described in [47]. Further details on panel fabrication, testing and FLUENT simulations are presented in $[10$, $11,2]$.

In the IGFEM simulations, we approximate the composite panel with a $2 \mathrm{D}$ do- 
main shown in Fig. 2b. An equivalent " $2 \mathrm{D}$ " conductivity defined as $\kappa_{2 \mathrm{D}}=\kappa t$ is used in order to account for the 2D approximation. Instead of a heat flux, a distributed heat source of strength $f(\mathbf{x})$ is applied in the interior of the domain. All sides of the domain are insulated except at the channel inlet, where $T_{\mathrm{in}}=22{ }^{\circ} \mathrm{C}$. If the Stefan-Boltzmann law is added to the heat equation (2), the resulting equation becomes nonlinear and has to be solved iteratively. Therefore, we linearize the Stefan-Boltzmann equation by assuming that $T$ is close to $T_{\mathrm{amb}}$ to obtain the following approximation

$$
q_{\mathrm{rad}} \approx-h_{\mathrm{rad}}\left(T-T_{\mathrm{amb}}\right),
$$

where $h_{\mathrm{rad}}=4 \epsilon \sigma_{B} T_{\mathrm{amb}}^{3}=5.6 \mathrm{Wm}^{-2} \mathrm{~K}^{-1}$. As a result, only a single convective heat loss term with $h=h_{\text {conv }}+h_{\text {rad }}$ is used in our IGFEM simulations.

The mesh used by FLUENT for a network of parallel channels shown in Fig. $2 \mathrm{c}$ contains a high concentration of elements in and near the channels to resolve the fluid velocities in the channel network. To achieve convergence in the average surface temperature and pressure drop, the number of elements needed exceeds 7 million. In contrast, the mesh for IGFEM in Fig. 2d contains only 9600 elements, is uniform everywhere and does not need to conform to the channel geometry. The maximum computational time (excluding the time to create the channel network) on a single thread is 1 minute for the bifurcating design with a $60 \times 80$ triangular mesh containing 9600 elements. In contrast, the mesh generation (excluding the creation of the channel geometry) in ANSYS and the FLUENT simulation take approximately seven hours to run on a single thread. Furthermore, creating a channel network consisting of lines is much simpler than creating a volumetric geometry of the channels.

The validation study was conducted for the parallel, bifurcating, spiral and serpentine channel networks shown in Figures 3a to 3d with total lengths of 1.54, 1.61, 1.46 and $1.35 \mathrm{~m}$, respectively. To obtain the temperature distribution using FLUENT, we simulate the channels with uniform rectangular cross sections of width 1.02 $\mathrm{mm}$ and height $0.84 \mathrm{~mm}$ corresponding to the average channel dimensions measured experimentally. Comparison of the top surface temperatures from FLUENT and the IGFEM temperature distribution in Fig. 3 shows excellent agreement. As shown in Table 2, the IGFEM results agree with FLUENT results within $1^{\circ} \mathrm{C}$. Moreoever, both simulations slightly underpredict the surface temperature measured experimentally as revealed in Fig. 3. In all cases, the difference between simulation and experiment temperatures is less than $2^{\circ} \mathrm{C}$, which is within the uncertainty of the experiments.

In Table 3, we compare the standard deviations of the temperature $\left(\sigma_{T}=\right.$ 
Table 2: Comparison of average temperatures $\langle T\rangle\left({ }^{\circ} \mathrm{C}\right)$ and maximum temperatures $T_{\max }\left({ }^{\circ} \mathrm{C}\right)$ from experiments, FLUENT and IGFEM, denoted respectively by the superscripts (E), (F) and (I). The top surface temperatures of experiments and FLUENT are used for comparison.

\begin{tabular}{|c|c|c|c|c|c|c|}
\hline Design & $\langle T\rangle^{(E)}$ & $\langle T\rangle^{(F)}$ & $\langle T\rangle^{(I)}$ & $T_{\max }^{(E)}$ & $T_{\max }^{(F)}$ & $T_{\max }^{(I)}$ \\
\hline Parallel & 30.4 & 29.0 & 28.7 & 37.2 & 36.5 & 36.8 \\
\hline Bifurcating & 29.8 & 28.8 & 28.4 & 34.0 & 33.0 & 32.9 \\
\hline Spiral & 30.0 & 28.4 & 28.2 & 33.2 & 31.5 & 31.4 \\
\hline Serpentine & 29.8 & 28.6 & 28.3 & 33.6 & 32.6 & 32.5 \\
\hline
\end{tabular}

$\left.\sqrt{\int_{\Omega}(T-\langle T\rangle)^{2} \mathrm{~d} \Omega /|\Omega|}\right)$ and the total pressure drop. All standard deviations agree within 10\%. The pressure drops calculated using the simplified models agree with experiments to within $11 \%$ while the FLUENT pressure drops differ from experiments by at most $20 \%$.

Table 3: Comparison of standard deviations of temperatures $\sigma_{T}\left({ }^{\circ} \mathrm{C}\right)$ and pressure drops $\Delta P(\mathrm{kPa})$ from experiments, FLUENT and IGFEM, denoted respectively by the superscripts (E), (F) and (I).

\begin{tabular}{|c|c|c|c|c|c|c|}
\hline Design & $\sigma_{T}^{(E)}$ & $\sigma_{T}^{(F)}$ & $\sigma_{T}^{(I)}$ & $(\Delta P)^{(E)}$ & $(\Delta P)^{(F)}$ & $(\Delta P)^{(I)}$ \\
\hline Parallel & 3.0 & 3.2 & 3.3 & 10.3 & 10.9 & 9.9 \\
\hline Bifurcating & 2.4 & 2.5 & 2.5 & 8.0 & 8.6 & 7.4 \\
\hline Spiral & 1.8 & 1.9 & 2.0 & 109 & 130 & 106 \\
\hline Serpentine & 2.5 & 2.1 & 2.3 & 110 & 121 & 97.8 \\
\hline
\end{tabular}

In order to simulate the pressure drop $\Delta P$, we use the reduced dimensions of the cross section, defined as the average minus the standard deviation of the experimentally measured cross-sectional dimensions $(0.94 \mathrm{~mm} \times 0.8 \mathrm{~mm})$. The reduced dimensions are used because (i) the smaller cross sections contribute significantly to the pressure drop, as evident by the strong dependence on the diameter in (16) and (ii) better agreement between experiments and simulations are obtained compared to that using the average dimensions. In the FLUENT simulations, the dynamics viscosity varies in the fluid according to (17). As shown in Table 3, the FLUENT simulations overestimate the pressure drops measured experimentally. However, due to the offseting effects of not accounting for the formation of the vortices at a branch or a bend in a channel $[48,22]$ and the use of the reduced dimensions, IGFEM slightly underestimates the pressure drops measured experimentally. 

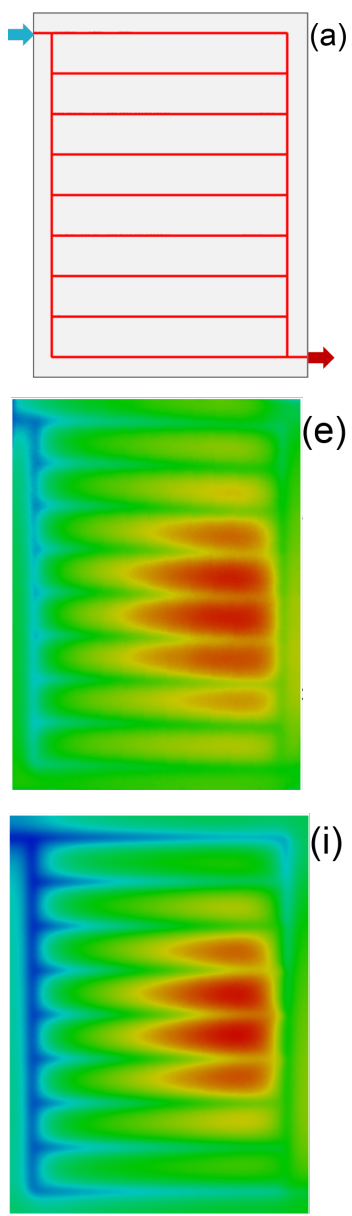

(i)
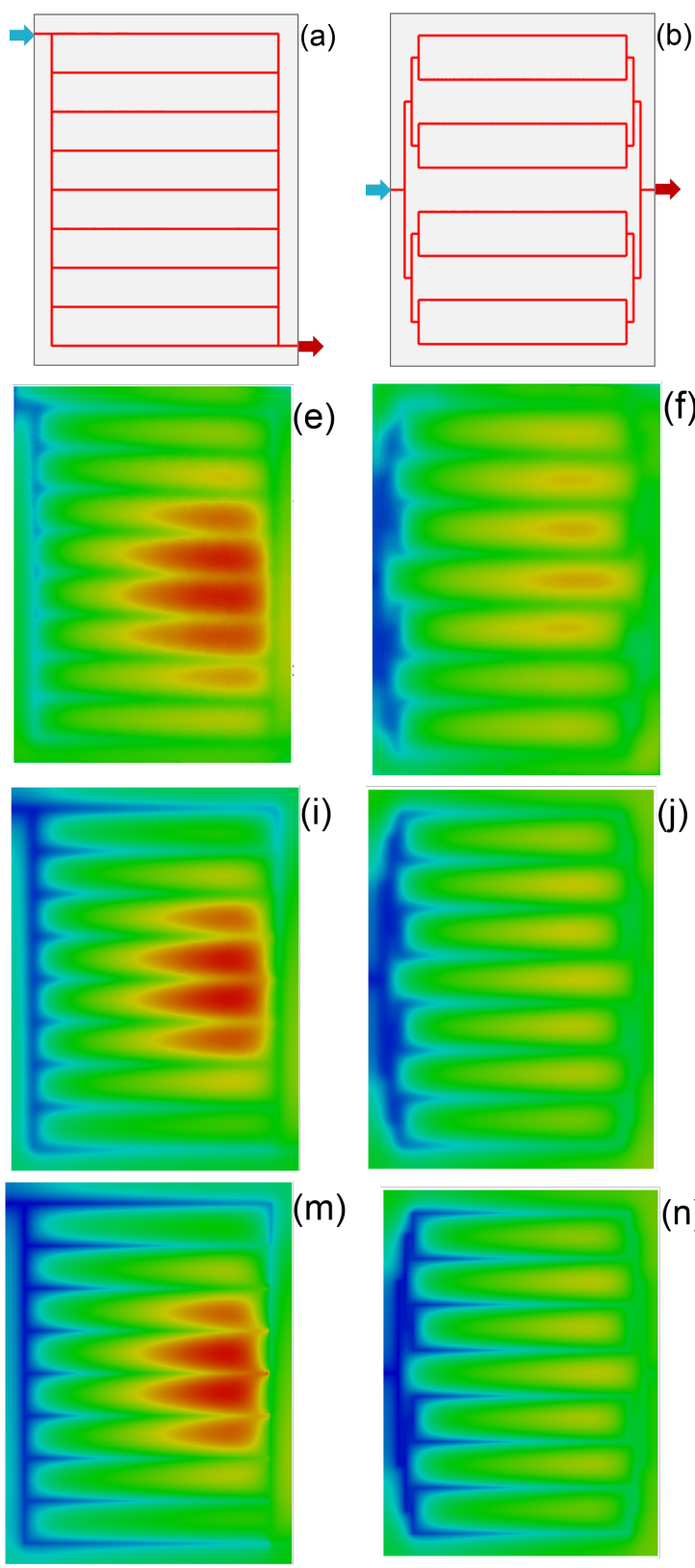

(f)

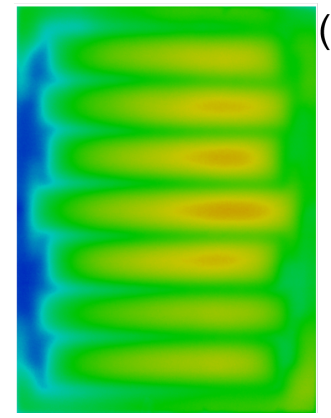

(m)

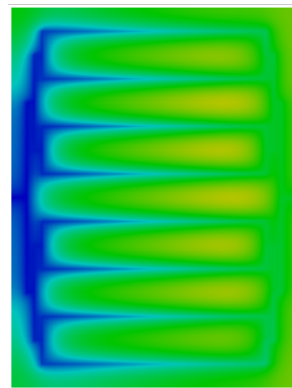

(b)
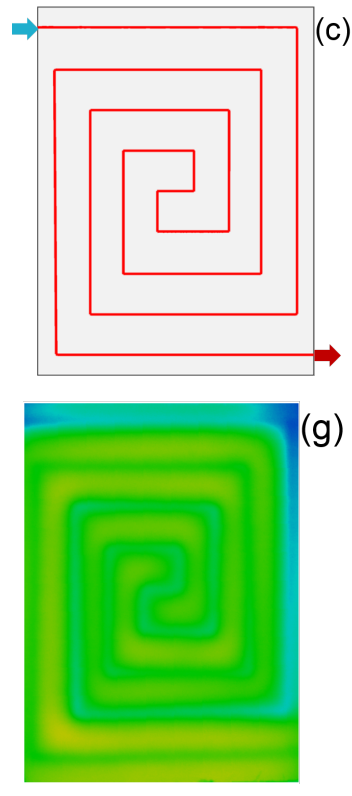

(g)

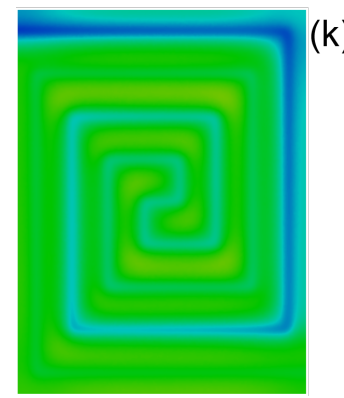

(n)

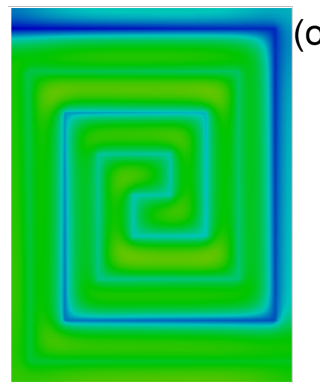

(o)

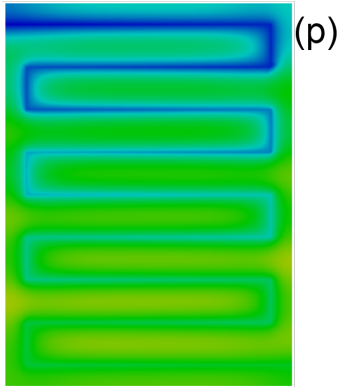

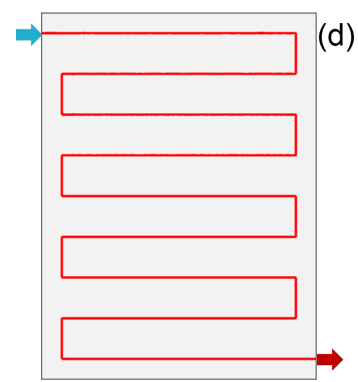

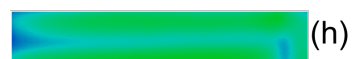

(h)

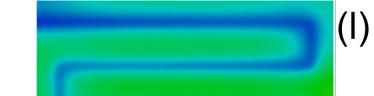

(I)

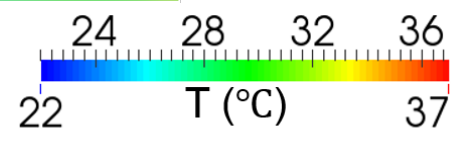

Figure 3: (a) Parallel, (b) bifurcating, (c) spiral and (d) serpentine reference designs for the cooling of composite panels. (e)-(h) Experiment and (i)-(l) FLUENT top surface temperature distributions compared to (m)-(p) IGFEM temperature solutions. 


\section{Adjoint Method and Sensitivity Analysis}

We now combine the simplified thermal model and associated IGFEM solver with gradient-based shape optimization algorithms available in MATLAB with the control points/end points of the channels as design variables. The interior-point [49] and SQP [50] algorithms are found to be sufficiently robust for this work. However, it is also found that the latter is more capable of producing solutions further from the initial guesses. As with all gradient-based optimization algorithms, we can only guarantee that a stationary solution (local minimum or saddle point) is found when certain conditions are met for a general well-posed optimization problem with nonlinear objective function and/or constraints [50]. To increase the chance of getting a solution close to the global optimum, we perform optimizations starting from many distinct initial designs.

The gradients are evaluated using a sensitivity analysis of the discretized equations based on an adjoint method developed recently for IGFEM [21]. Some of the basic steps of the sensitivity analysis directly relevant to the thermal problem of interest are described hereafter. Details on the evaluation of terms such as the derivatives of the shape functions with respect to design parameters and the design velocity field can be found in [21].

Let us denote the nodal coordinates of the mesh by $\mathbf{X}$ and the design parameter vector by $\mathbf{d}=\left\{d_{1}, \ldots, d_{n_{d}}\right\}^{\prime}$. The general form of the optimization problem is

$$
\begin{aligned}
\min _{\mathbf{d}} & \theta(T(\mathbf{X}(\mathbf{d}), \mathbf{d}), \mathbf{X}, \mathbf{d}), \\
\text { such that } & \mathbf{g}(T(\mathbf{X}(\mathbf{d}), \mathbf{d}), \mathbf{X}, \mathbf{d}) \leq 0, \\
\text { and } & {[K(\mathbf{X}(\mathbf{d}), \mathbf{d})]\{T(\mathbf{X}(\mathbf{d}), \mathbf{d})\}=\{F(\mathbf{X}(\mathbf{d}), \mathbf{d})\} . }
\end{aligned}
$$

In this work, for reasons discussed later in Section 6.2, we consider the following specific form of the objective function:

$$
\theta=|\Omega|\left(T_{p}\right)^{p}=\int_{\Omega} T^{p} \mathrm{~d} \Omega=\int_{\Omega}\left(\{N\}^{\prime}\{T\}\right)^{p} \mathrm{~d} \Omega,
$$

where $|\Omega|$ is the area of the domain in $2 \mathrm{D}$ and $T_{p}=\left(\int_{\Omega} T^{p} \mathrm{~d} \Omega /|\Omega|\right)^{1 / p}$ is the $p$ mean of temperature. For a given $p$, the optimization of the $p$-norm defined as $\|T\|_{p}=\left(\int_{\Omega} T^{p} \mathrm{~d} \Omega\right)^{1 / p}$ is equivalent to that of the $p$-mean.

Without loss of generality, we restrict the following discussion to a single design parameter $d$. Given a function $h(\mathbf{x}, d)$ and the shape velocity field $\mathbf{v}(\mathbf{x}, d)=\partial \mathbf{x} / \partial d$, where $\mathbf{x}$ denotes the spatial coordinates, the total shape material derivative is defined as $\stackrel{*}{h}=\left(\nabla_{x} h\right)^{\prime} \mathbf{v}+\partial h / \partial d$. Using the Reynolds transport formula and the chain rule 
$\frac{*}{h_{1} h_{2}}=h_{1} h_{2}^{*}+\stackrel{*}{h_{1}} h_{2}$, the derivative of $(20)$ with respect to the design parameter $d$ is given by

$$
\begin{aligned}
\frac{\partial \theta}{\partial d}= & \int_{\Omega_{s}}\left[\frac{*}{\left(\{N\}^{\prime}\{T\}\right)^{p}}+\left(\{N\}^{\prime}\{T\}\right)^{p} \nabla_{x} \cdot \boldsymbol{v}\right] \mathrm{d} \Omega \\
= & \int_{\Omega_{s}}\left[p\left(\{N\}^{\prime}\{T\}\right)^{p-1} \frac{*}{\{N\}^{\prime}\{T\}}+\left(\{N\}^{\prime}\{T\}\right)^{p} \nabla_{x} \cdot \boldsymbol{v}\right] \mathrm{d} \Omega \\
= & \int_{\Omega_{s}}\left[p\left(\{N\}^{\prime}\{T\}\right)^{p-1}\left(\left\{\frac{\partial N}{\partial d}\right\}^{\prime}\{T\}+\{N\}^{\prime}\left\{T^{*}\right\}\right)\right. \\
& \left.+\left(\{N\}^{\prime}\{T\}\right)^{p} \nabla_{x} \cdot \boldsymbol{v}\right] \mathrm{d} \Omega .
\end{aligned}
$$

In writing $\{\stackrel{*}{N}\}$ as $\{\partial N / \partial d\}$, we have implicitly assumed that all the arguments of $\{N\}$ have been expressed explicitly as functions of $d$. Note that, unlike for the standard FEM, the term $\{\partial N / \partial d\} \neq 0$ in XFEM/GFEM/IGFEM [21]. The term, easily missed if the non-discretized form of (20) is differentiated directly, cannot be neglected to obtain the correct derivative.

Relation (21) can be rewritten as

$$
\frac{\partial \theta}{\partial d}=\left\{F_{\text {adj }}\right\}^{\prime}\{\stackrel{*}{T}\}+R
$$

where

$$
\left\{F_{\text {adj }}\right\}=\int_{\Omega_{s}} p\left(\{N\}^{\prime}\{T\}\right)^{p-1}\{N\} \mathrm{d} \Omega
$$

and

$$
R=\int_{\Omega_{s}}\left[p\left(\{N\}^{\prime}\{T\}\right)^{p-1}\left\{\frac{\partial N}{\partial d}\right\}^{\prime}\{T\}+\left(\{N\}^{\prime}\{T\}\right)^{p} \nabla_{x} \cdot \boldsymbol{v}\right] \mathrm{d} \Omega .
$$

To obtain $\{\stackrel{*}{T}\}$, we differentiate the discretized weak form of the thermal equations to obtain

$$
[K]\{\stackrel{*}{T}\}=\left\{F_{\text {pseudo }}\right\}=-\left[\frac{\partial K}{\partial d}\right]\{T\}+\left\{\frac{\partial F}{\partial d}\right\},
$$


where

$$
\begin{aligned}
{\left[\frac{\partial K}{\partial d}\right]=} & \int_{\Omega_{s}}\left(\left[\frac{\partial B}{\partial d}\right][\kappa][B]^{\prime}+[B][\kappa]\left[\frac{\partial B}{\partial d}\right]^{\prime}+h\left\{\frac{\partial W}{\partial d}\right\}\{N\}^{\prime}+h\left\{\frac{\partial N}{\partial d}\right\}\{W\}^{\prime}\right. \\
+ & \left.\left([B][\kappa][B]^{\prime}+h\{W\}\{N\}^{\prime}\right) \nabla_{x} \cdot \boldsymbol{v}\right) \mathrm{d} \Omega \\
+ & \sum_{i=1}^{n_{c h}} \int_{\Gamma_{f}^{(i)}}\left[\left\{\frac{\partial W}{\partial d}\right\}\left([B] \gamma^{(i)}\left\{t^{(i)}\right\}\right)^{\prime}+\{W\}\left([B] \gamma^{(i)}\left\{t^{(i)}\right\}\right)^{\prime} \nabla_{\partial} \cdot \boldsymbol{v}\right. \\
+ & \left.\{W\}\left(\left[\frac{\partial B}{\partial d}\right] \gamma^{(i)}\left\{t^{(i)}\right\}+[B] \frac{\partial \gamma^{(i)}}{\partial d}\left\{t^{(i)}\right\}+[B] \gamma^{(i)}\left\{\frac{\partial t^{(i)}}{\partial d}\right\}\right)^{\prime}\right] \mathrm{d} \Gamma
\end{aligned}
$$

and

$$
\begin{aligned}
\left\{\frac{\partial F}{\partial d}\right\} & =\int_{\Omega_{s}}\left(\left\{\frac{\partial W}{\partial d}\right\}\left(f+h T_{\mathrm{amb}}\right)+\{W\} f^{*}+\{W\}\left(f+h T_{\mathrm{amb}}\right) \nabla_{x} \cdot \boldsymbol{v}\right) \mathrm{d} \Omega \\
& +\int_{\Gamma_{q}}\left(\left\{\frac{\partial V}{\partial d}\right\} q_{p s}^{\prime \prime}+\{V\} q_{p s}^{\prime \prime}+\{V\} q_{p s}^{\prime \prime} \nabla_{\partial} \cdot \boldsymbol{v}\right) \mathrm{d} \Gamma .
\end{aligned}
$$

Here, we have used $\nabla_{\partial}$. to denote the surface divergence (or line divergence in 2D), i.e, $\nabla_{\partial} \cdot \mathbf{h}=\operatorname{tr}\left[\left(\nabla_{x} \mathbf{h}\right)^{\prime}(\mathbf{I}-\mathbf{n n})\right]$, where the $j$ th column of $\nabla_{x} \mathbf{h}$ is the spatial gradient of $h_{j}$. The evaluation of $\left\{\partial t^{(i)} / \partial d\right\}$ is straightforward and will not be detailed here, and we describe the calculation of $\{\partial W / \partial d\}$ and $\partial \gamma^{(i)} / \partial d$ in the Appendix II. The boundary term of (27) vanishes as $q_{p s}^{\prime \prime}=0$ in the present work.

Let the subscripts $f$ and $p s$ respectively denote the free and prescribed nodal values of the temperature field. $\{\stackrel{*}{T}\}$ can be partitioned into two parts, i.e.,

$$
\{\stackrel{*}{T}\}=\left\{\begin{array}{l}
\stackrel{*}{T}_{f} \\
\stackrel{*}{T}_{p s}
\end{array}\right\}=\left\{\begin{array}{c}
\stackrel{*}{T}_{f} \\
0
\end{array}\right\},
$$

Similarly, by partitioning $[K]$ into four parts, (25) becomes

$$
\left[K_{f f}\right]\left\{\stackrel{*}{T}_{f}\right\}=\left\{F_{\text {pseudo, } f}\right\} \text {. }
$$

At this point, we can already calculate (21) by solving (29) for each design parameter. If $n_{f} \times n_{f}$ is the size of $\left[K_{f f}\right]$ and $n_{d}$ is the number of design parameters, 
then the number of operations is $O\left(n_{d} n_{f}^{2}\right)$. The number of operations can be made independent of $n_{d}$ by using the adjoint method, in which the product of a multiplier $\left\{\Lambda_{f}\right\}$ and (29) is added to (21) to yield

$$
\begin{aligned}
\frac{\partial \theta}{\partial d} & =\left\{F_{a d j}\right\}^{\prime}\{\stackrel{*}{T}\}+R+\left\{\Lambda_{f}\right\}^{\prime}\left(-\left[K_{f f}\right]\left\{\stackrel{*}{T}_{f}\right\}+\left\{F_{\text {pseudo }, f}\right\}\right) \\
& =\left(\left\{F_{a d j, f}\right\}^{\prime}-\left\{\Lambda_{f}\right\}^{\prime}\left[K_{f f}\right]\right)\left\{\stackrel{*}{T}_{f}\right\}+\left\{\Lambda_{f}\right\}^{\prime}\left\{F_{\text {pseudo }, f}\right\}+R .
\end{aligned}
$$

Upon setting the coefficient of $\left\{\stackrel{T}{T}_{f}^{*}\right.$ to zero, we have

$$
\left[K_{f f}\right]^{\prime}\left\{\Lambda_{f}\right\}=\left\{F_{\mathrm{adj}, f}\right\}
$$

and

$$
\frac{\partial \theta}{\partial d}=\left\{\Lambda_{f}\right\}^{\prime}\left\{F_{p s e u d o, f}\right\}+R .
$$

It should be noted that (31) needs to be solved only once regardless of $n_{d}$. We also emphasize that $\left[K_{f f}\right]$ is not symmetric in general due to the convective term.

\section{Optimization of Parallel Network Designs}

In the remainder of this paper, we apply the shape optimization method to the parallel channel designs with different number of branches. Parallel designs with two, five and eight branches are presented in Fig. 4a, 4e and $4 \mathrm{~g}$ together with the nonconforming structured mesh used for the optimization analysis. The problem setup is similar to that of Fig. $2 \mathrm{~b}$ except for the nature of the thermal loading applied

to the battery cooling panel: To more accurately simulate the thermal load that a battery cooling panel would receive, no heat loss from the surface is assumed and a uniform heat source of $500 \mathrm{Wm}^{-2}$ is applied. This represents the scenario where a panel is heated from both sides. The thermal conductivity of the composite is raised to $2.7 \mathrm{Wm}^{-1} \mathrm{~K}^{-1}$ to represent a composite with a higher fiber volume fraction of $60 \%$, the inlet temperature is set at $27{ }^{\circ} \mathrm{C}$ and the inlet flow rate is $28.2 \mathrm{ml} / \mathrm{min}$ unless specified otherwise. The width and height of the channel cross section are set to 0.75 $\mathrm{mm}$. These conditions are representative of the heat flux, coolant temperature and coolant flow rate found in Chevy Volt cooling panels [16]. As indicated earlier, the design parameters are the locations of all control points indicated by blue diamonds in the figure, except the inlet and outlet control points, which are fixed. Thus the shape optimization problems described in Figures 4a, 4e and 4g involve 12, 24 and 36 design parameters, respectively. The bounding boxes for all design parameters are chosen as (in m) $\{0.005 \leq x \leq 0.145,0.005 \leq y \leq 0.195\}$. 


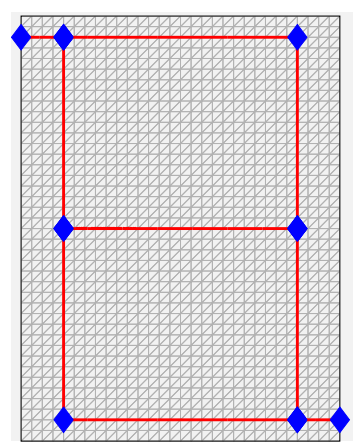

(a)

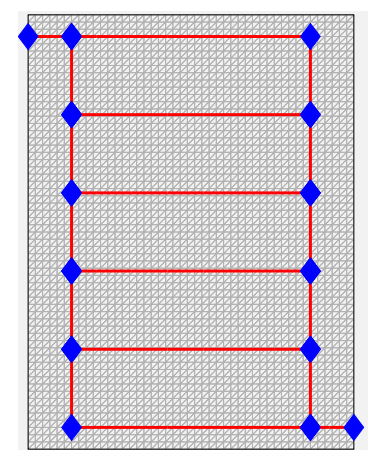

(e)

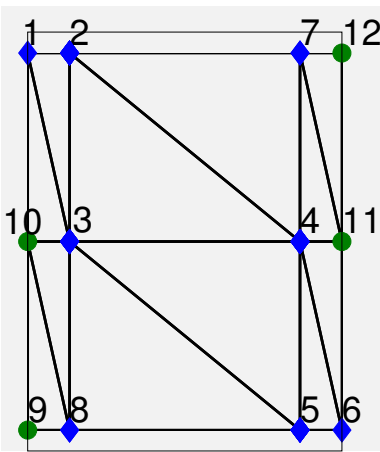

(b)

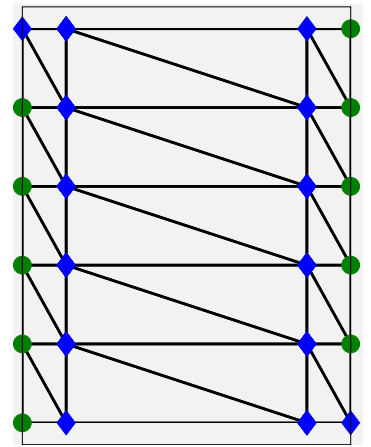

(f)

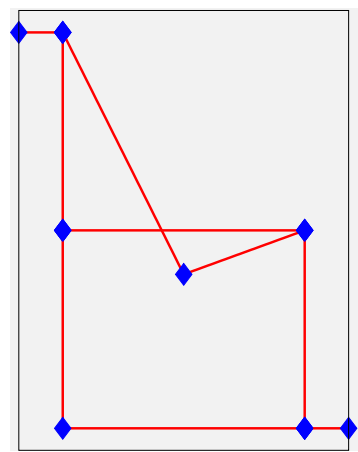

(c)

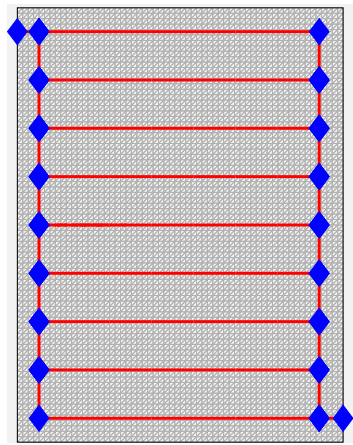

$(\mathrm{g})$

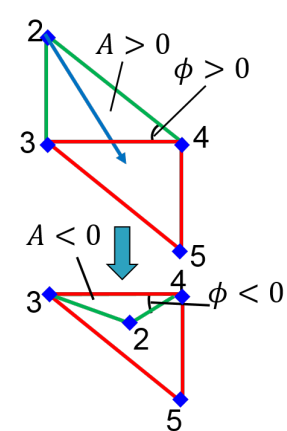

(d)

Figure 4: (a) two-branch, (e) five-branch and (g) eight-branch parallel networks used as reference designs for comparison with optimal designs. (b), (f), (h) Corresponding nonlinear constraint triangles used to help prevent self-intersections as explained in Section 6.1. Examples of (c) selfcrossing channel network and (d) nonlinear constraint violation.

\subsection{Geometrical constraints}

One of the key constraints of the design problem is associated with the "selfcrossing" of some of the channels, which renders the problem unphysical, as illustrated in Fig. 4c. To address this issue, we apply a set of simple geometrical constraints to triangles constructed from the networks as shown in Fig. 4b, $4 \mathrm{f}$ and $4 \mathrm{~h}$. Let $\phi$ and $A$ respectively denote the interior angle and area of a triangle such as $\{2,3,4\}$ in Fig. $4 d$. The geometrical constraints take the form: (i) $\sin \phi \geq \sin \phi_{o}$ for some $\phi_{o} \in[0, \pi / 2]$ (typically, $\phi_{o}=0.5^{\circ}$ ) and (ii) $A \geq A_{o}$ (typically, $A_{o}=0.001 \times$ area of domain). Mathematically, condition (ii) is redundant. However, numerically, it is needed when two vertices are very close together and calculation of $\sin \phi$ becomes inaccurate. The derivative of the above nonlinear constraints with respect to the design parameters can readily be found as the vertex locations are directly related 
to the design parameters.

\subsection{Choice of $p$ in $p$-mean}

As described earlier, the max function $T_{\max }(\mathbf{d})=\max _{\mathbf{x}} T(\mathbf{x}, \mathbf{d})$ is not differentiable in the classical sense [34], and is typically replaced by a differentiable alternative such as the Kreisselmeier-Steinhauser function or the $p$-norm $/ p$-mean in stress-based optimization [35, 36, 33]. Adopting the latter approach, we replace $T_{\max }(\mathbf{d})$ with $T_{p}$, noting that $\lim _{p \rightarrow \infty} T_{p}=T_{\max }(\mathbf{d})$ since $T(\mathbf{x}, \mathbf{d})$ is a continuous function of $\mathbf{x}$ and the domain is bounded and closed [51]. In general, $T_{p} \leq T_{\max }(\mathbf{d}) \leq\|T\|_{p}[51,52]$. However, there is no explicit relationship between $T_{\max }(\mathbf{d})$ and $T_{p}$.

The choice of $p$ is the result of a compromise: large values may cause $T_{p}$ to be ill-conditioned or less smooth [33, 36], but small values may not allow $T_{p}$ to capture reliably the trend in $T_{\max }(\mathbf{d})$, resulting in local regions of high temperature similar to regions of stress concentration in structural optimization problems [33, 36]. Based on our numerical observtions and on recommended values for stress-based optimization [33], we adopt $p=8$. It is worth mentioning here that to integrate (20) exactly over a triangle with $p=8$, a 16-point Gauss-Dunavant quadrature [53] is required.

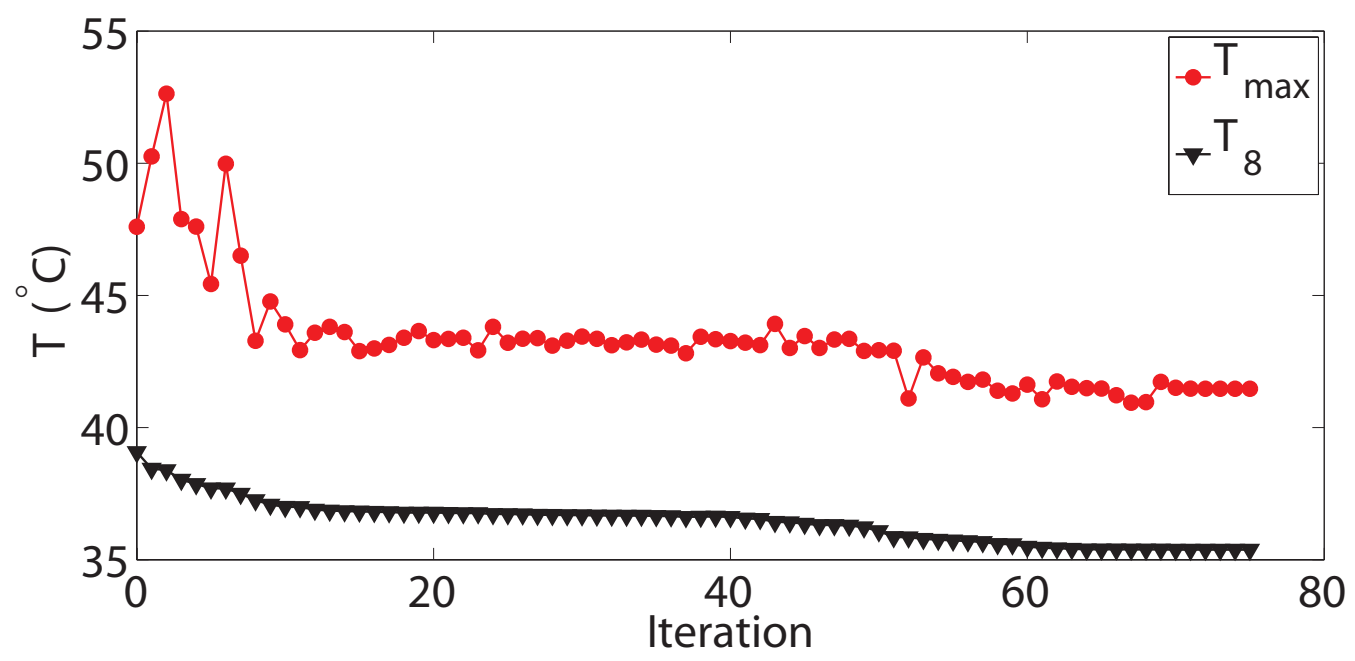

Figure 5: Optimization history of the five-branch parallel design.

Fig. 5 illustrates the optimization history of $T_{8}$ and $T_{\max }$ for the five-branch parallel design study starting from the reference design, showing how $T_{\max }$ follows the overall downward trend of $T_{8}$. 


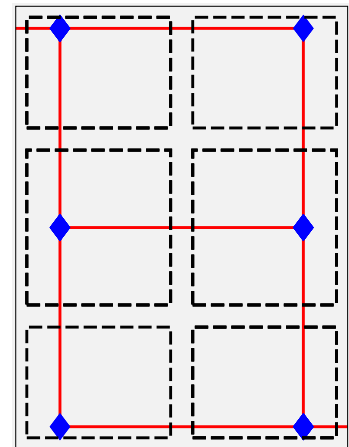

(a)

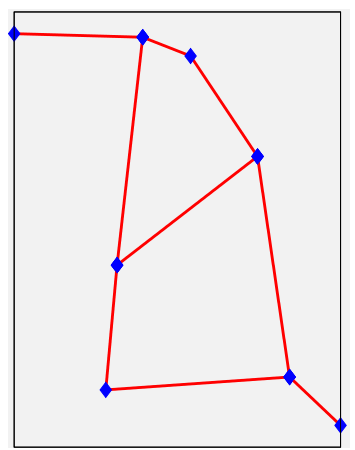

(b)

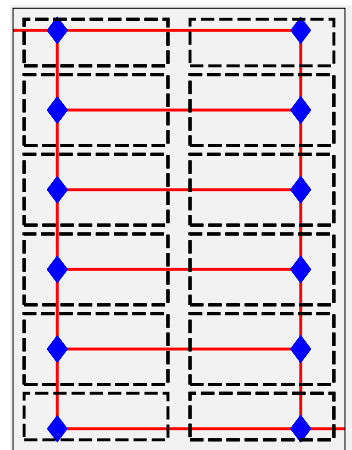

(c)

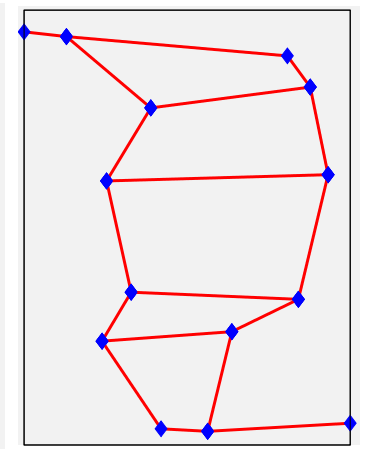

(d)

Figure 6: Bounding boxes indicated by dash rectangles for generating random initial designs for (a) two and (c) five branches together with examples of initial designs ((b) and (d)).

\subsection{Optimal designs of branched networks}

A wide range of potential optimal configurations is investigated by starting the optimization from more than 40 distinct designs for each number of branches. The initial designs are obtained by randomizing the initial positions of the control points within non-overlapping bounding boxes shown in Fig. 6a and 6c. Some of the initial designs are presented in Fig. 6b and 6d.

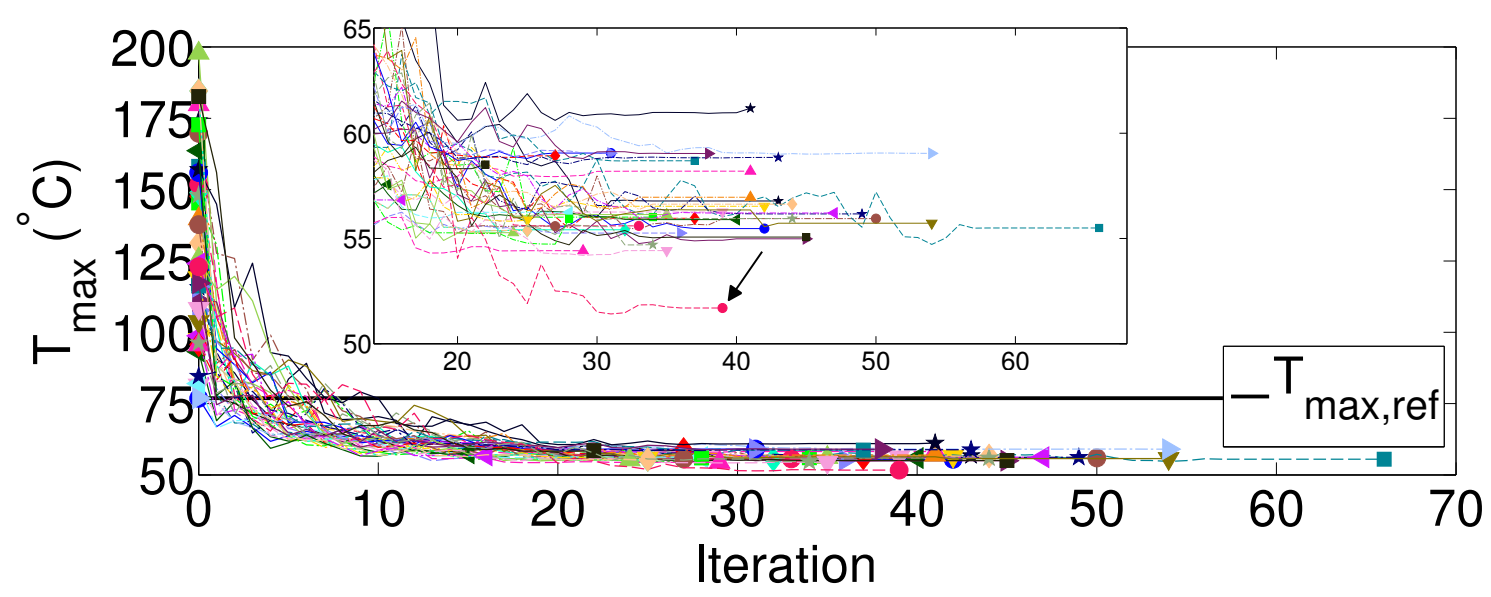

Figure 7: Evolution of $T_{\max }$ during the shape optimization of the two-branch case. The inset presents a zoomed-in view of the maximum temperature associated with multiple local optimal configurations.

As apparent from the optimization histories for the two-branch case shown in Fig. 
7, the optimizer reduces $T_{\max }$ by more than $20^{\circ} \mathrm{C}$ compared to the reference maximum temperature $T_{\max , \text { ref }}$ (represented by a horizontal line in the figure). Numerous optimal solutions are obtained, as shown in the inset by the various stationary values of $T_{\max }$. It should be noted that, due to round-off errors and various approximations in the algorithm (such as the approximate Hessian [50, 49]), optimal solutions with similar shapes may have slightly different objective function values and design parameter values, as also observed in [16] with the SQP algorithm in MATLAB. The "best optimal" configuration (in terms of $T_{\max }$ ) corresponds to the red circular point indicated by an arrow in Fig. 7 with $T_{\max }=51.7^{\circ} \mathrm{C}$, and is shown in Fig. 8b.

In Fig. 8, we present the optimal designs for two, five and eight branches, together with the corresponding reference designs and temperature distributions. As apparent from these figures, the thermal performance of the optimal designs is greatly superior to that of the reference designs in terms of both maximum temperature and temperature uniformity. The predicted configuration depends strongly on the number of branches in the network: the optimal designs for two to six branches tend to adopt a more "vertical configuration" while those with seven to twelve branches appear to be diagonally oriented. For a low number of branches, it is reasonable to stretch the channels as long as possible to maximize area coverage. Hence, the vertically oriented design is preferred. However, the higher the number of branches, the lower the flow rate in each channel, which results in a more rapid temperature rise along the channel. Therefore, the optimal designs for high number of branches tend to have shorter channel segments, i.e., the diagonal orientation is preferred. As shown in Fig. 9a, the optimal designs tend to equalize the flow rates in the interior channels, thereby achieving a more uniform temperature field. This observation is consistent with that of an existing study [24], where it is shown that the "distributor" designs with the most uniform flow distribution generally have the best performance in terms of maximum temperature difference and thermal resistance.

Fig. 9b shows that the reduction in the maximum temperature of the microvascular panel diminishes as the number of branches increases and saturates between eight to ten branches. As a reference, we have also plotted the expected rise in coolant temperature, $\Delta T_{\text {coolant }}=Q /\left(\dot{m} c_{f}\right)$ if all the applied heat is absorbed by the coolant. This rise in temperature is also a lower bound for $\Delta T$. At ten branches, the maximum temperature of the optimal design is only $1.5^{\circ} \mathrm{C}$ above $\Delta T_{\text {coolant }}$. We also observe that the pressure drop needed to circulate the coolant is substantially higher for the vertically oriented optimal designs with six branches or less. The pressure drop appears to be the lowest for the eight branch design, beyond which $\Delta P$ increases slightly with the number of branches. 


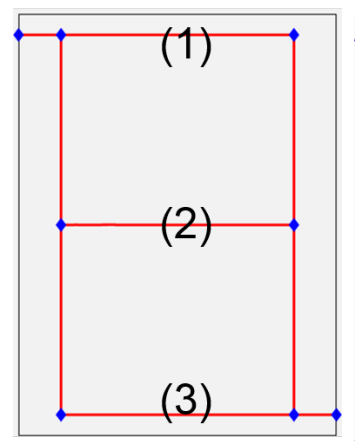

(a)

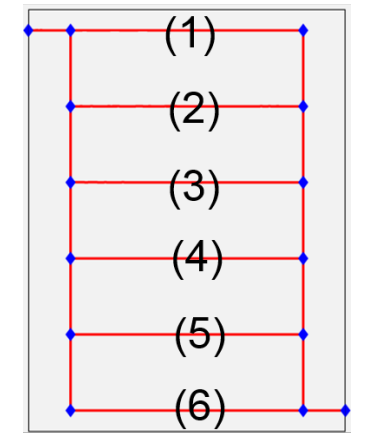

(d)

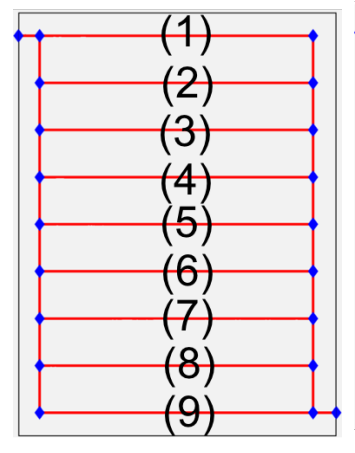

(g)

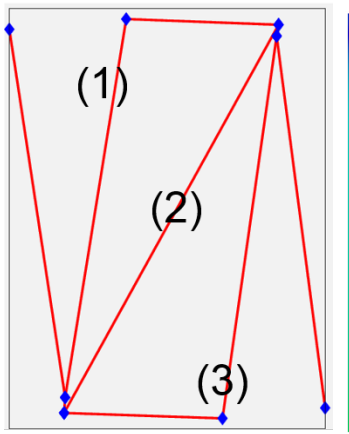

(b)

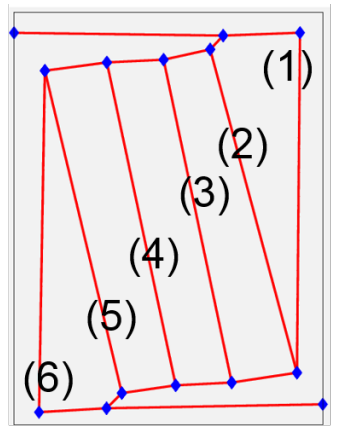

(e)

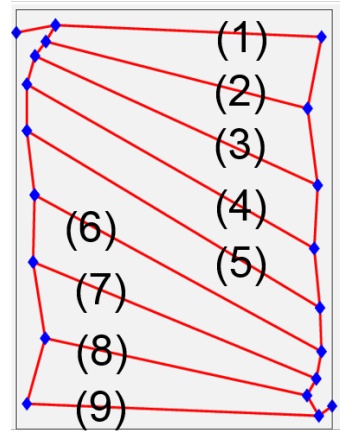

(h)
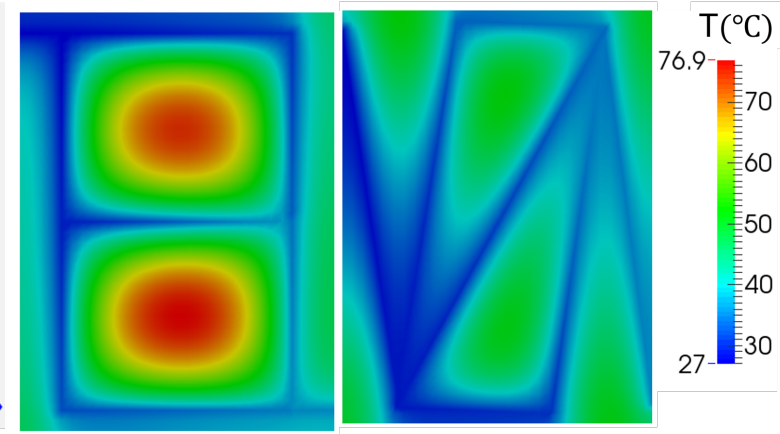

(c)
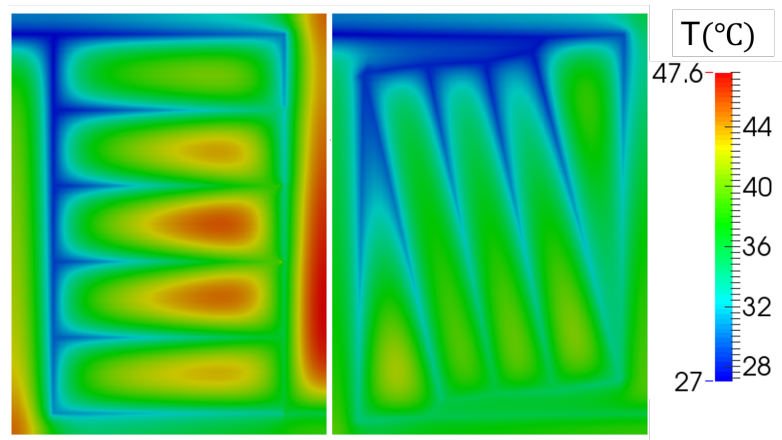

(f)
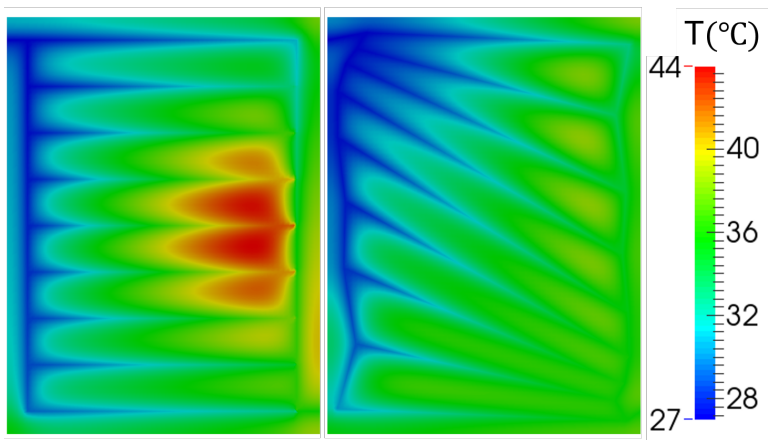

(i)

Figure 8: Reference (a, d, g) and optimal (b, e, h) designs for two, five and eight branches. (c), (f) and (i): Temperature distributions corresponding to the optimal designs compared with the reference temperature distributions with the upper bound of the color bar set to the maximum temperature of the corresponding reference design. 


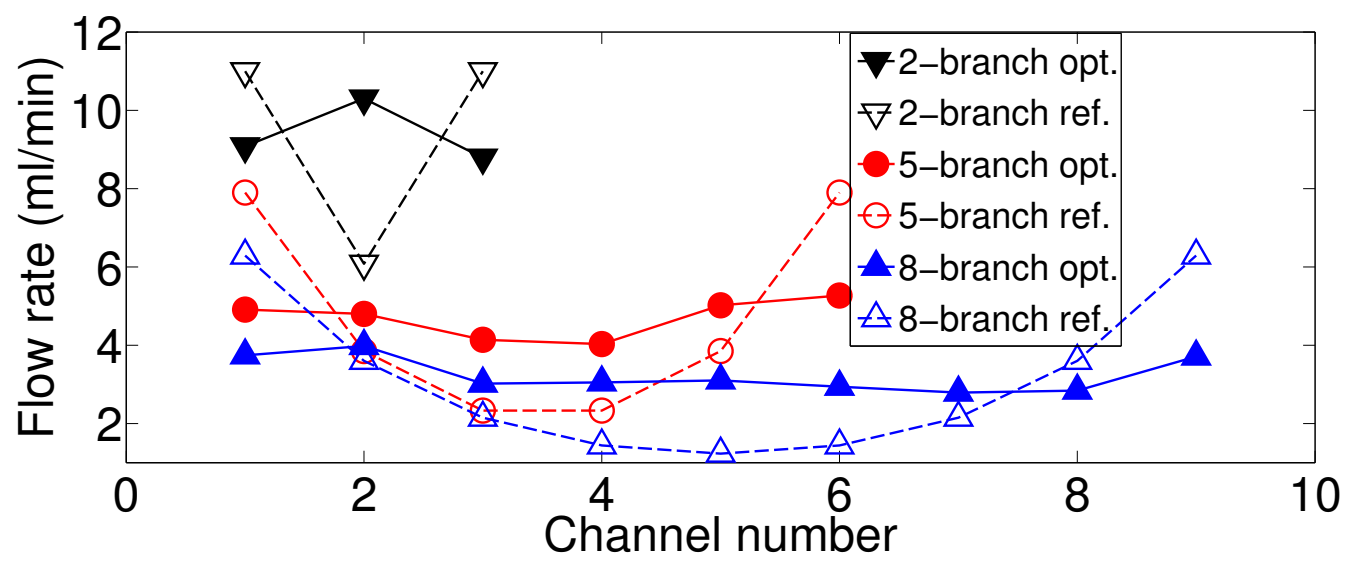

(a)

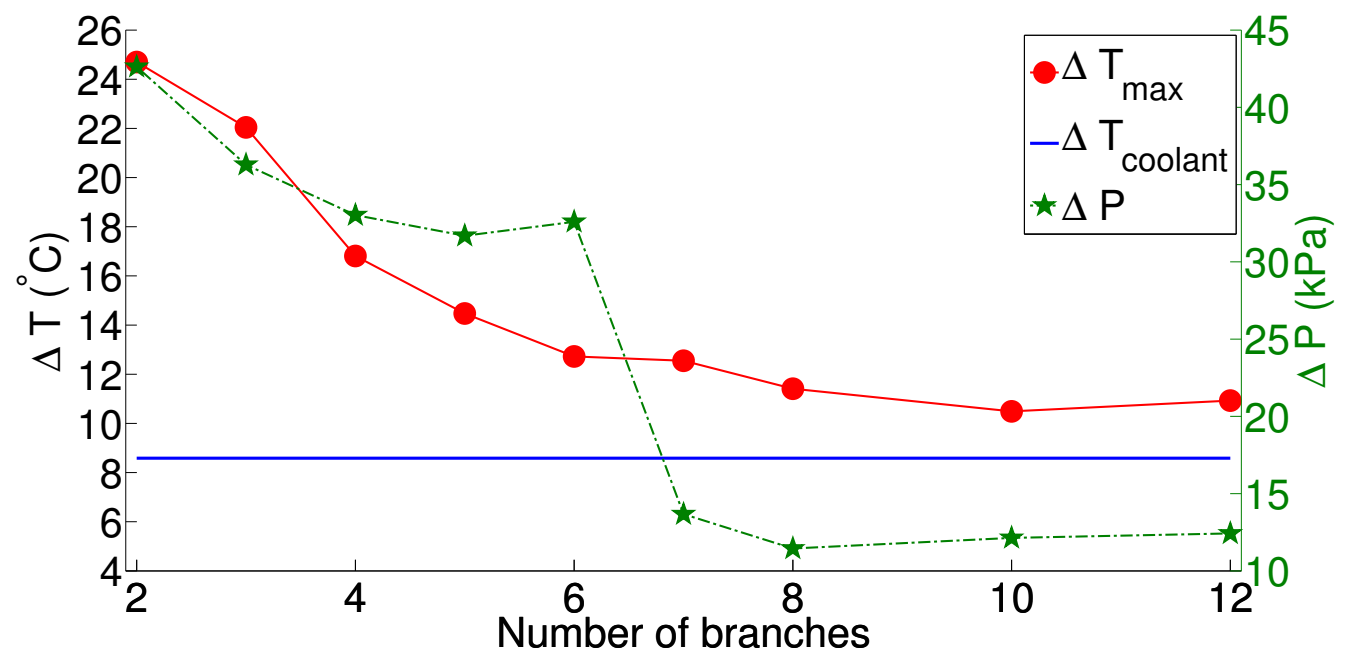

(b)

Figure 9: (a) Flow rates in the interior channels of the two-, five-, and eight-branch reference and optimal designs, with the channel numbers shown in Fig. 8. (b) Temperature difference $\Delta T=$ $T_{\max }-T_{\mathrm{in}}$ and pressure drop $\Delta P$ obtained for the optimal designs as a function of number of branches. The temperature rise of the coolant $\Delta T_{\text {coolant }}$ is also shown for comparison.

\subsection{Effect of flow rate on optimal design}

All the design results presented thus far are based on a constant flow rate of 28.2 $\mathrm{ml} / \mathrm{min}$. In this section, the effect of the flow rate on the design of the five-branch network is investigated by varying the flow rate from 2.82 to $56.3 \mathrm{ml} / \mathrm{min}$. Some of the optimal designs are shown in Fig. 10a to 10d. The transition from a diagonal design to a vertical design between 11.3 and $16.9 \mathrm{ml} / \mathrm{min}$ is consistent with the earlier 


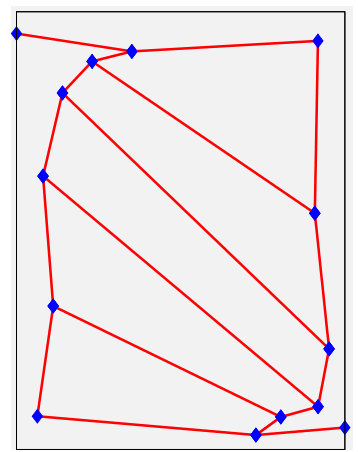

(a)

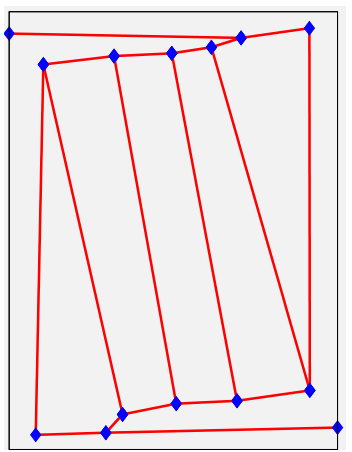

(b)

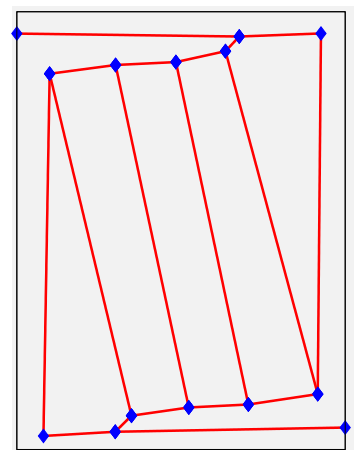

(c)

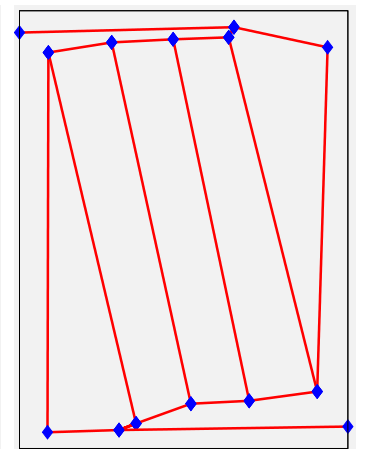

(d)

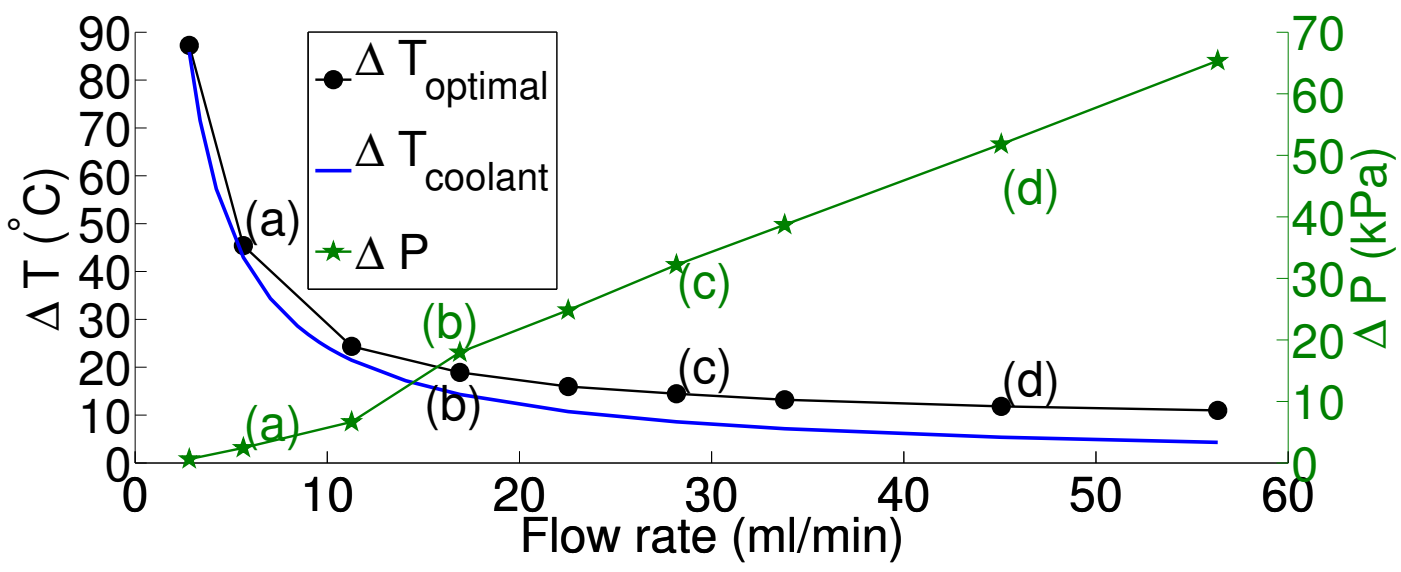

(e)

Figure 10: Best optimal configurations for flow rates of (a) 5.63, (b) 16.9, (c) 28.2 and (d) 45.1 $\mathrm{ml} / \mathrm{min}$. Fig. (e) presents the effect of the flow rate on the temperature difference $\Delta T=T_{\max }-T_{\text {in }}$ and pressure drop $\Delta P$ associated with the best optimal design. The temperature rise of the coolant $\Delta T_{\text {coolant }}$ is also shown for comparison.

observations: for low flow rates, the channels tend to be shorter to maximize their cooling effect.

As evident in Fig. 10e, the thermal impact of the embedded network increases (i.e., $\Delta T=T_{\max }-T_{\text {in }}$ decreases) with increasing flow rate, but at a declining rate in a trend consistent with previous studies $[17,54,55]$. This trend is due to the positive correlation between $\Delta T$ and the temperature rise of the coolant $\Delta T_{\text {coolant }}$, itself linked to the flow rate through $\Delta T_{\text {coolant }} \sim Q /\left(\dot{m} c_{f}\right)$. The "cost" of circulating the coolant is illustrated through the evolution of the pressure drop $\Delta P$, which, as expected, increases with the flow rate. However, it shows two distinct linear regions, with a transition corresponding to the flow rate at which the aforementioned transition from 
vertical to diagonal configurations takes place.

\subsection{Validation of five-branch optimal design}

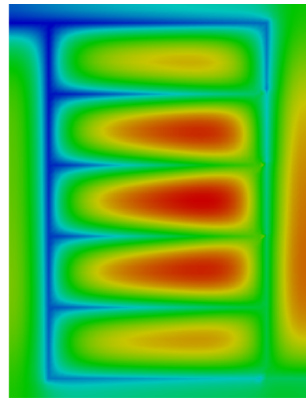

(a)

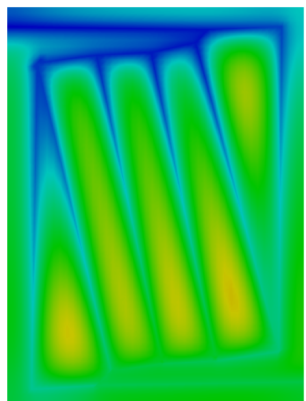

(b)

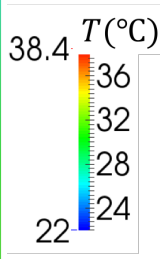

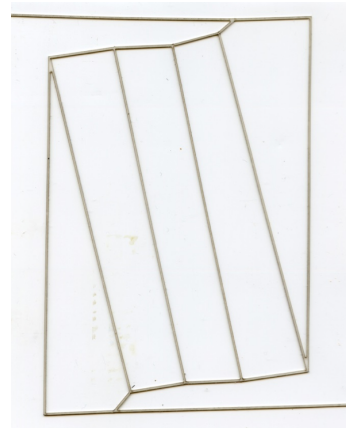

(c)

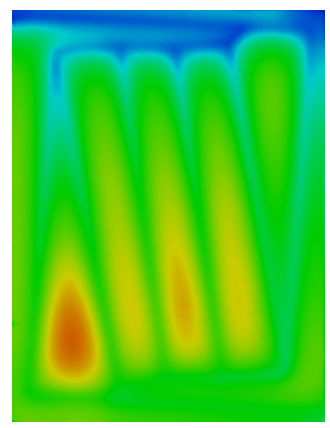

(d)

Figure 11: Numerical temperature distributions of (a) the reference and (b) the optimal five-branch networks. (c) Laser-cut sacrificial template used to embed the optimal five-branch network in the composite panel for the validation study. (d) Experimentally-measured temperature distribution of the optimal network.

In this final section, we present a validation study of the five-branch optimal design under the thermal loading conditions described in the validation exercise of Section 4 and for the same flow rate of $28.2 \mathrm{ml} \mathrm{min}^{-1}$. As shown in Fig. 11a and 11b, the IGFEM temperature distribution of the optimal network is significantly more uniform than that of the reference network. The average and maximum temperatures of the optimal network are respectively $28.8^{\circ} \mathrm{C}$ and $34.6{ }^{\circ} \mathrm{C}$ compared to $30.8{ }^{\circ} \mathrm{C}$ and $38.4{ }^{\circ} \mathrm{C}$ for the reference network. For this validation study, we have fabricated the optimal network by using the sacrificial template shown in Fig. 11c [10, 11, 2]. The experimentally measured average and maximum temperatures are respectively $30.5{ }^{\circ} \mathrm{C}$ and $36.5{ }^{\circ} \mathrm{C}$, which are slightly higher than the simulated values, but within experimental error. The experimental standard deviation of $2.5^{\circ} \mathrm{C}$ agrees closely with that of simulated value of $2.87^{\circ} \mathrm{C}$. On the other hand, the experimental pressure drop of $26.4 \mathrm{kPa}$ is higher than the simulated value of $20.8 \mathrm{kPa}$, which is calculated using the hydraulic equation and the experimentally measured minimum cross section dimensions. One possible source of the discrepancy is the omission of additional pressure penalty at the corners of the channels in the hydraulics equations.

\section{Conclusions}

A simplified thermal/hydraulic model has been used to model the impact of an embedded channel network on the thermal field in a thin microvascular composite and 
the pressure drop needed to circulate the coolant. To facilitate the discretization of the many configurations analyzed in the design process, and to avoid issues associated with mesh distortion present in conventional finite-element-based shape optimization studies, an interface-enriched generalized finite element method (IGFEM) has been used for the thermal solver, allowing for accurate and efficient solutions of the thermal problem with meshes that do not conform to the network configurations. A SUPG scheme has been introduced to stabilize the IGFEM solution. The IGFEM solver has been verified against nonlinear, fluid/thermal 3D FLUENT solutions, and validated against experimental measurements. By combining the thermal IGFEM solver with a gradient-based shape optimization scheme, we have optimized the designs of parallel networks defined by a set of branched channels. To avoid self-intersection of the channels, a set of simple nonlinear constraints have been incorporated in the design problem formulation, and each design analysis has involved multiple initial configurations to address the presence of a large number of local optima.

The shape optimization studies have led to configurations substantially different from the reference parallel channel configurations, with thermal performance greatly improved both in terms of reducing the maximum temperature and achieving a uniform temperature field. The predicted optimal designs have shown shorter and longer channel segments for low and high number of branches, respectively. Consistent with the previous trend, we also found a transition from a design with shorter channels at low flow rates to one with longer channels for higher flow rates. Furthermore, we have demonstrated that the simulation results of the five-branch optimal design agree with experimental results. The method described here can be applied to a wide range of objective functions and constraints.

\section{Acknowledgement}

This work has been supported by the DEMS Program of the NSF Division of Civil, Mechanical and Manufacturing Innovation (Award No. 1436720); the Air Force Office of Scientific Research National Defense Science and Engineering Graduate (NDSEG) Fellowship (32 CFR 168a); the Center for Electrical Energy Storage funded by DOE Office of Basic Energy Sciences (Grant No. 615 DOE ANL 9F-31921). The authors wish to thank Dr. Masoud Safdari for useful discussions.

\section{Appendix I}

Since $T_{m}$ is always lower than $T_{w}$, the simplified model tends to underpredict the temperature in the microvascular composite. We can estimate the difference as follows: Using (1), the definition of the Nusselt number $\mathrm{Nu}=h_{o} D_{h} / \kappa_{f}$ and 
$q^{\prime}=4 A h_{o}\left(T_{w}-T_{m}\right) / D_{h}$, where $h_{o}, D_{h}, \kappa_{f}$ respectively denote the convection coefficient between the wall and the fluid, the hydraulic diameter of the channel and conductivity of the fluid, one can show that $T_{w}-T_{m}=D_{h}^{2} \dot{m} c_{f} /\left(4 A \kappa_{f} \mathrm{Nu}\right) \mathrm{d} T_{m} / \mathrm{d} s$. Given a net heat transfer $Q$ into a channel of length $L, \mathrm{~d} T_{m} / \mathrm{d} s \approx Q /\left(\dot{m} c_{f} L\right)$ and

$$
T_{w}-T_{m} \approx \frac{D_{h}^{2}}{4 A} \frac{Q}{\kappa_{f} \mathrm{Nu} L} .
$$

For fully developed flows, $\mathrm{Nu}$ and hence $T_{w}-T_{m}$ do not depend on $\dot{m}$. Based on the parameters in Section 4 and denoting the kinematic viscosity by $\nu$, typical values of the Reynolds number and Prandtl number are $\operatorname{Re}=u_{\text {ave }} D_{h} / \nu=185$ and $\operatorname{Pr}=26$, respectively. This yields a thermal entry length $\left(0.05 \operatorname{Re} \operatorname{Pr} D_{h}\right)$ of approximately 0.2 $\mathrm{m}$ compared to a total channel length, $L \approx 1.5 \mathrm{~m}$. Using $\mathrm{Nu} \approx 3.66$ for rectangular tubes with aspect ratio equal to that of the channels in Section 4, and constant axial wall heat flux and peripheral wall temperature boundary condition [45], the difference between $T_{w}$ and $T_{m}$ is estimated to be approximately $1.6{ }^{\circ} \mathrm{C}$.

\section{Appendix II}

The derivative of the weighing function with respect to a design parameter is given by

$$
\begin{aligned}
\left\{\frac{\partial W}{\partial d}\right\} & =\left\{\frac{\partial N}{\partial d}\right\}+\frac{1}{2} \sum_{i=1}^{n_{c h}}\left(\frac{\partial h_{e}^{(i)}}{\partial d}[B]\left\{t^{(i)}\right\}\right. \\
& \left.+h_{e}^{(i)}\left[\frac{\partial B}{\partial d}\right]\left\{t^{(i)}\right\}+h_{e}^{(i)}[B]\left\{\frac{\partial t^{(i)}}{\partial d}\right\}\right),
\end{aligned}
$$

where

$$
\frac{\partial h_{e}^{(i)}}{\partial d}=-\frac{h_{e}^{(i) 2}}{2} \sum_{j=1}^{n_{n}} \operatorname{sign}\left(\left\{B_{j}\right\}^{\prime}\left\{t^{(i)}\right\}\right)\left(\left\{\frac{\partial B_{j}}{\partial d}\right\}^{\prime}\left\{t^{(i)}\right\}+\left\{B_{j}\right\}^{\prime}\left\{\frac{\partial t^{(i)}}{\partial d}\right\}\right),
$$

and

$$
\left\{B_{j}\right\}=\left\{\frac{\partial N_{j}}{\partial x}, \frac{\partial N_{j}}{\partial y}\right\}^{\prime}
$$

To obtain $\partial \gamma^{(i)} / \partial d=c_{f} \partial \dot{m}^{(i)} / \partial d$, we first need to obtain $\partial P_{j} / \partial d$, i.e, the derivative of the nodal pressures with respect to a design parameter. Differentiating the hydraulics equation $[G]\{P\}=\{S\}$, we have

$$
[G]\left\{\frac{\partial P}{\partial d}\right\}=-\left[\frac{\partial G}{\partial d}\right]\{P\}+\left\{\frac{\partial S}{\partial d}\right\},
$$


which is reminiscent of the pseudo-force equation (25). If nodes $j, k$ are the end nodes of channel $i$, the derivative of its mass flow rate is

$$
\frac{\partial \dot{m}^{(i)}}{\partial d}=g^{(i)} \operatorname{sign}\left(P_{j}-P_{k}\right)\left(\frac{\partial P_{j}}{\partial d}-\frac{\partial P_{k}}{\partial d}\right) .
$$

\section{References}

[1] A. P. Esser-Kahn, P. R. Thakre, H. Dong, J. F. Patrick, V. K. Vlasko-Vlasov, N. R. Sottos, J. S. Moore, S. R. White, Three-dimensional microvascular fiberreinforced composites, Advanced Materials 23 (2011) 3654-3658.

[2] R. C. R. Gergely, S. J. Pety, B. P. Krull, J. F. Patrick, T. Q. Doan, A. M. Coppola, P. R. Thakre, N. R. Sottos, J. S. Moore, S. R. White, Multidimensional vascularized polymers using degradable sacrificial templates, Advanced Functional Materials 25 (2015) 1043-1052.

[3] D. Therriault, S. R. White, J. A. Lewis, Chaotic mixing in three-dimensional microvascular networks fabricated by direct-write assemble, Nature Materials 2 (2003) 265-271.

[4] K. S. Toohey, N. R. Sottos, J. A. Lewis, J. S. Moore, S. R. White, Self-healing materials with microvascular networks, Nature Materials 6 (2007) 581-585.

[5] J. Patrick, K. Hart, B. P. Krull, C. E. Diesendruck, J. S. Moore, S. R. White, N. R. Sottos, Continuous self-healing life-cycle in vascularized structural composites, Advanced Materials 26 (2014) 4302-4308.

[6] B. D. Kozola, L. A. Shipton, V. K. Natrajan, K. T. Christensen, S. R. White, Characterization of active cooling and flow distribution in microvascular polymers, J Intel. Mat. Syst. Str. 21 (2010) 1147-1156.

[7] D. M. Phillips, M. R. Pierce, J. W. Baur, Mechanical and thermal analysis of microvascular networks in structural composite panels, Composites: Part A 42 (2011) 1609-1619.

[8] A. R. Najafi, A. Coppola, S. Soghrati, N. R. Sottos, S. R. White, P. H. Geubelle, Microvascular composite skin panels for hypersonic aircraft, in: AIAA SciTech, 2014. 
[9] A. Coppola, N. Sottos, S. R. White, Thermomechanical characterization of actively cooled vascularized composites, in: Proceedings of the Society of Engineering Science 51st Annual Technical Meeting, 2014.

[10] S. J. Pety, N. R. Sottos, S. R. White, Actively cooled battery packaging using microvascular composites, in: Proc. of the 19th Inter. Conf. on Composite Materials (ICCM 19), 2013.

[11] S. Pety, M. H. Y. Tan, A. R. Najafi, P. H. Geubelle, N. Sottos, S. R. White, Microvascular composites for intergrated battery packaging and cooling, in: Proceedings of the Society of Engineering Science 51st Annual Technical Meeting, 2014.

[12] A. A. Pesaran, Battery thermal mangement in EVs and HEVs, in: Advanced Automotive Battery Conference, 2001.

[13] G. H. Kim, A. A. Pesaran, Battery thermal management system design modeling, in: 22nd International Battery, Hybrid and Fuel Cell Electric Vehicle Conference and Exhibition, 2006.

[14] J. Obradovic, S. Boria, G. Belingardi, Lightweight design and crash analysis of composite frontal impact energy absorbing structures, Composite Structures 94 (2012) 423-430.

[15] Dirk H. -J.A. Lukaszewicz, in:, A. Elmarakbi (Ed.), Advanced Composite Materials for Automotive Applications: Structural Integrity and Crashworthiness, John Wiley and Sons, 2014, pp. 99-127.

[16] A. Jarrett, I. Y. Kim, Design optimization of electric vehicle battery cooling plates, J. Power Sources 196 (2011) 10359-10368.

[17] J. C. Kurnia, A. P. Sasmito, A. S. Mujumdar, Numerical investigation of laminar heat transfer performance of various cooling channel designs, Applied Thermal Engineering 31 (2011) 1293-1304.

[18] S. Soghrati, A. M. Aragón, C. A. Duarte, P. H. Geubelle, An interface-enriched generalized FEM for problems with discontinuous gradient fields, Int. J. Numer. Methods Eng. 89 (2012) 991-1008.

[19] S. Soghrati, P. H. Geubelle, A 3D interface-enriched generalized finite element method for weakly discontinuous problems with complex internal geometries, Comput. Methods Appl. Mech. Eng. 217-220 (2012) 46-57. 
[20] N. H. Kim, Y. Chang, Eulerian shape design sensitivity analysis and optimization with a fixed grid, Comput. Methods Appl. Mech. Eng. 194 (2005) 32913314 .

[21] A. R. Najafi, M. Safdari, D. A. Tortorelli, P. H. Geubelle, A gradient-based shape optimization scheme using an interface-enriched generalized FEM, Comput. Methods Appl. Mech. Eng. 296 (2015) 1-17.

[22] S. M. Senn, D. Poulikakos, Laminar mixing, heat transfer and pressure drop in tree-like microchannel nets and their application for thermal management in polymer electrolyte fuel cells, J. Power Sources 130 (2004) 178-191.

[23] S. M. Baek, S. H. Yu, J. H. Nam, C.-J. Kim, A numerical study on uniform cooling of large-scale PEMFCs with different coolant flow field designs, Applied Thermal Engineering 31 (2011) 1427-1434.

[24] B. Ramos-Alvarado, P. Li, H. Liu, A. Hernandez-Guerrero, CFD study of liquidcooled heat sinks with microchannel flow field configurations for electronics, fuel cells and concentrated solar cells, Applied Thermal Engineering 31 (2011) 24942507.

[25] S. Soghrati, P. R. Thakre, S. R. White, N. R. Sottos, P. H. Geubelle, Computational modeling and design of actively-cooled microvascular materials, Int. J. Heat Mass Transfer 55 (2012) 5309-5321.

[26] T. Matsumori, T. Kondoh, A. Kawamoto, T. Nomura, Topology optimization for fluid-thermal interaction problems under constant input power, Struct Multidisc Optim 47 (2013) 571.

[27] A. M. Aragón, J. K. Wayer, P. H. Geubelle, D. E. Goldberg, S. R. White, Design of microvascular flow networks using multi-objective genetic algorithms, Comput. Methods Appl. Mech. Eng. 197 (2008) 4399-4410.

[28] A. M. Aragón, K. J. Smith, P. H. Geubelle, S. R. White, Multi-physics design of microvascular materials for active cooling applications, J. Computational Physics 230 (2011) 5178-5198.

[29] A. M. Aragón, R. Saksena, B. D. Kozola, P. H. Geubelle, K. T. Christensen, S. R. White, Multi-physics optimization of three-dimensional microvascular polymeric components, J. Computational Physics 233 (2013) 132-147. 
[30] A. Bejan, S. Lorente, Constructal theory of generation of configuration in nature and engineering, J. Applied Physics 100 (2006) 041301.

[31] J. H. Ryu, D. Choi, S. Kim, Three-dimensional numerical optimization of a manifold microchannel heat sink, Int. J. Heat Mass Transfer 46 (2003) 15531562 .

[32] A. Husain, K.-Y. Kim, Shape optimization of microchannel heat sink for microelectronics cooling, IEEE Transactions on Components and Packaging Technologies 31 (2008) 322-330.

[33] C. Le, J. Norato, T. Bruns, C. Ha, D. Tortorelli, Stress-based topology optimization for continua, Struct. Multidisc. Optim. 41 (2010) 605-620.

[34] F. H. Clarke, Optimization and nonsmooth analysis, Society for Industrial and Applied Mathematics, 1990, Ch. 2, p. 47.

[35] R. J. Yang, C. J. Chen, Stress-based topology optimization, Structural Optimization 12 (1996) 98-105.

[36] P. Duysinx, O. Sigmund, New developments in handling stress constraints in optimal material distribution, American Institute of Aeronautics and Astronautics 98-4906 (1998) 1501-1509.

[37] S. Soghrati, A. R. Najafi, J. H. Lin, K. M. Hughes, S. R. White, N. R. Sottos, P. H. Geubelle, Computational analysis of actively-cooled 3D woven microvascular composites using a stabilized interface-enriched generalized finite element method, Int. J. Heat Mass Transfer 65 (2013) 153-164.

[38] A. M. Aragón, C. A. Duarte, P. H. Geubelle, Generalized finite element enrichment functions for discontinuous gradient fields, Int. J. Numer. Methods Eng. 82 (2009) 242-268.

[39] M. H. Y. Tan, M. Safdari, A. R. Najafi, P. H. Geubelle, A NURBS-based interface-enriched generalized finite element scheme for the thermal analysis and design of microvascular composites, Comput. Methods Appl. Mech. Eng. 283 (2015) 1382-1400.

[40] W. M. Kays, M. E. Crawford, Convective heat and mass transfer, McGraw-Hill, 1993. 
[41] A. N. Brooks, T. J. R. Hughes, Streamline upwind/Petrov-Galerkin formulations for convection dominated flow with particular emphasis on the incompressible Navier-Stokes equation, Comput. Methods Appl. Mech. Eng. 32 (1982) 199-259.

[42] Q.-G. Ning, T.-W. Chou, Closed-form solutions of the in-plane effective thermal conductivities of woven-fabric composites, Composite Science and Technology 55 (1995) 41-48.

[43] C. A. Brebbia, A. J. Ferrante, Computational Hydraulics, Butterworths, 1983.

[44] M. Bahrami, M. Yovanovich, J. R. Culham, Pressure drop of fully-developed, laminar flow in microchannel of arbitrary cross-section, J. Fluids Eng. 128 (2006) $1036-1044$.

[45] R. K. Shah, A. L. London, Laminar Flow Forced Convection in Ducts, Academic Press, 1978, Ch. 2 and 7, p. 20 and 200.

[46] A. Jarrett, Multi-objective design optimization of electric vehicle battery cooling plates considering thermal and pressure objective functions, Master's thesis, Queen's University (2011).

[47] R. Rolfes, U. Hammerschmidt, Transverse thermal conductivity of CFRP laminates: a numerical and experimental validation of approximation formulae, Composite Science and Technology 54 (1995) 45-54.

[48] D. Haller, P. Woias, N. Kockmann, Simulation and experimental investigation of pressure lost and heat transfer in microchannel networks containing bends and T-junctions, Int. J. Heat Mass Transfer 52 (2009) 2678-2689.

[49] R. H. Byrd, J. C. Gilbert, J. Nocedal, A trust region method based on interior point techniques for nonlinear programming, Mathematical Programming 89 (2000) 149-185.

[50] P. T. Boggs, J. W. Tolle, Sequential quadratic programming, Acta Numerica 4 (1995) 1-51.

[51] W. Rudin, Real and Complex Analysis, McGraw-Hill, 1987, Ch. 3, pp. 61-71.

[52] G. B. Folland, Real Analysis, John Wiley and Sons, 1999, Ch. 6, p. 186.

[53] D. A. Dunavant, High degree efficient symmetrical Gaussian quadrature rules for the triangle, Int. J. Numer. Methods Eng. 21 (1985) 1129-1148. 
[54] A. M. Coppola, A. S. Griffin, N. R. Sottos, S. R. White, Retention of mechanical performance of polymer matrix composites above the glass transition temperature by vascular cooling, Composites Part A: Applied Science and Manufacturing 78 (2015) 412-423.

[55] A. M. Coppola, L. Hu, P. R. Thakre, M. Radovic, I. Karaman, N. R. Sottos, S. R. White, Active cooling of a microvascular shape memory alloy-polymer matrix composite hybrid material, Advanced Engineering Materials. 